\title{
Rogue waves and downshifting in the presence of damping
}

\author{
A. Islas and C. M. Schober \\ Department of Mathematics, University of Central Florida, Orlando, FL, USA
}

Received: 2 October 2010 - Revised: 6 December 2010 - Accepted: 13 December 2010 - Published: 9 February 2011

\begin{abstract}
Recently Gramstad and Trulsen derived a new higher order nonlinear Schrödinger (HONLS) equation which is Hamiltonian (Gramstad and Trulsen, 2011). We investigate the effects of dissipation on the development of rogue waves and downshifting by adding an additonal nonlinear damping term and a uniform linear damping term to this new HONLS equation. We find irreversible downshifting occurs when the nonlinear damping is the dominant damping effect. In particular, when only nonlinear damping is present, permanent downshifting occurs for all values of the nonlinear damping parameter $\beta$. Significantly, rogue waves do not develop after the downshifting becomes permanent. Thus in our experiments permanent downshifting serves as an indicator that damping is sufficient to prevent the further development of rogue waves. We examine the generation of rogue waves in the presence of damping for sea states characterized by JONSWAP spectrum. Using the inverse spectral theory of the NLS equation, simulations of the NLS and damped HONLS equations using JONSWAP initial data consistently show that rogue wave events are well predicted by proximity to homoclinic data, as measured by the spectral splitting distance $\delta$. We define $\delta_{\text {cutoff }}$ by requiring that $95 \%$ of the rogue waves occur for $\delta<\delta_{\text {cutoff. We find }}$ that $\delta_{\text {cutoff }}$ decreases as the strength of the damping increases, indicating that for stronger damping the JONSWAP initial data must be closer to homoclinic data for rogue waves to occur. As a result when damping is present the proximity to homoclinic data and instabilities is more crucial for the development of rogue waves.
\end{abstract}

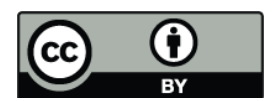

Correspondence to: C. M. Schober (cschober@mail.ucf.edu)

\section{Introduction}

Some theoretical models attribute the development of rogue waves in deep water to a nonlinear focusing of uncorrelated waves in a very localized region of the sea. This focusing may be related to the BenjaminFeir (BF) instability, described to leading order by the nonlinear Schrödinger (NLS) equation (Henderson et al., 1999; Kharif and Pelinovsky, 2001, 2004). Homoclinic orbits of modulationally unstable solutions of the NLS equation which undergo large amplitude excursions away from their target solution exhibit many of the observed properties of rogue waves and can be used to model rogue waves (Osborne et al., 2000; Calini and Schober, 2002).

A more accurate description of the water wave dynamics is obtained by retaining higher order terms in the asymptotic expansion for the surface wave displacement. A commonly used higher order NLS equation is the Dysthe equation (Dysthe, 1979). Homoclinic orbits of the unstable Stokes wave have been shown to be robust to the higher order corrections in the Dysthe equation. Laboratory experiments conducted in conjunction with numerical simulations of the Dysthe equation established that the generic long-time evolution of initial data near an unstable Stokes wave with two or more unstable modes is chaotic (Ablowitz et al., 2000, 2001). Subsequent studies of the Dysthe equation showed that for a rather general class of such initial data, the modulational instability leads to high amplitude waves, structurally similar to the optimal phase modulated homoclinic solutions of the NLS equation, rising intermittently above a chaotic background (Calini and Schober, 2002; Schober, 2006). These earlier studies relating homoclinic solutions and rogue waves ignored the fact that the Dysthe equation is not Hamiltonian and neglected damping which, even when weak, can have a significant effect on the wave dynamics.

Published by Copernicus Publications on behalf of the European Geosciences Union. 
Recently Gramstad and Trulsen (2011) brought the Dysthe equation into Hamiltonian form obtaining a new higher order nonlinear Schrödinger (HONLS) equation (Gramstad and Trulsen, 2011). In this paper we investigate the effects of dissipation on the development of rogue waves and downshifting in one space dimension by adding a nonlinear damping term (the $\beta$-term which damps the mean flow Kato and Oikawa, 1995) and a uniform linear damping term to this new HONLS equation. The governing equations and their properties are set up in Sect. 2.

Downshifting in the evolution of nearly uniform plane waves was first observed by Lake et al. (1977). Their experiment showed that after the wavetrain becomes strongly modulated, it recurs as a nearly uniform wavetrain with the dominant frequency permanently downshifted. Permanent downshifting has been confirmed in other laboratory experiments on the evolution of the Stokes wave ( $\mathrm{Su}$, 1982; Huang et al., 1996). Conservative models have been inadequate in describing permanent downshifting and several damped models have been suggested, e.g. where the damping is modeling wave breaking or eddy viscosity to capture downshifting (Trulsen and Dysthe, 1990; Hara and Mei, 1991, 1994).

Our previous studies of rogue waves show that an $\epsilon$ neighborhood of the unstable plane wave (the same regime in which Lake examined downshifting) is effectively a rogue wave regime for the Dysthe equation since the likelihood of obtaining a rogue wave is extremely high. We reexamine this issue in Sect. 3 using the new HONLS equation. In Sect. 4, using initial data for an unstable modulated wavetrain we examine whether irreversible downshifting (although here downshifting of the wave number is considered) occurs in our damped HONLS equation and what characterizes the damped wave train evolution on a short and long time scale.

We find that rogue waves may emerge in both the linear and nonlinear damped regimes that were not present without damping. Although damping decreases the growth rates of the individual modes, the modes may coalesce due to changes in their focusing times, thus resulting in larger waves in the damped regime. Even so, on average, the strength is smaller and fewer rogue waves occur when damping is present. While downshifting is not expected in the linearly damped case, we examine the effect of linear damping on the development of rogue waves due to its widespread use in modeling dissipative processes.

The nonlinear $\beta$-term models damping of the mean flow and since it is large only near the crest ofthe envelope, the damping is localized when the wavetrain is strongly modulated. Due to the BF instability, irreversible downshifting occurs when the nonlinear damping is the dominant damping effect. In particular, when only nonlinear damping is present, permanent downshifting occurs for all values of the nonlinear damping parameter $\beta$, appearing abruptly for larger values of $\beta$. Significantly, we find that after permanent downshifting occurs, rogue waves do not appear in the nonlinearly damped evolution. Thus in our experiments permanent downshifting serves as an indicator that there has been sufficient cumulative damping to inhibit the further development of rogue waves.

Developing sea states, where nonlinear wave-wave interactions continue to occur, are described by the Joint North Sea Wave Project (JONSWAP) power spectrum. A spectral quantity, the splitting distance $\delta$ between simple periodic points of the Floquet spectrum of the associated AKNS spectral problem of the initial condition may be used to measure the proximity in spectral space to unstable waves and homoclinic data of the NLS equation. In (Islas and Schober, 2005; Schober, 2006), simulations of both the NLS and Dysthe equations using JONSWAP initial data consistently show that rogue wave events are well predicted by proximity to homoclinic data, as measured by $\delta$.

In Sect. 5 we examine the generation of rogue waves in the presence of damping for sea states characterized by JONSWAP spectrum. Using the damped HONLS equation we find that both the strength and likelihood of rogue waves occuring in a given simulation are typically smaller when damping is present. We define $\delta_{\text {cutoff }}$ by requiring that $95 \%$ of the rogue waves occur for $\delta<\delta_{\text {cutoff. }}$ Significantly, we find that $\delta_{\text {cutoff }}$ is generally decreasing as the strength of the damping increases. Thus when damping is present the JONSWAP initial data must be closer to instabilities and homoclinic data for rogue waves to occur. The proximity to homoclinic data and instabilities becomes more essential for the development of rogue waves when damping is present.

\section{Analytical background}

\subsection{A new damped higher order nonlinear Schrödinger equation}

The equations for inviscid deep water waves, as well as the nonlinear Schrödinger (NLS) equation, can be derived from a Lagrangian. Ideally, a higher order NLS equation would retain this feature. The commonly used higher order NLS equation (Dysthe, 1979), often referred to as Dysthe's equation, is not Hamiltonian and does not conserve momentum. Recently, Gramstad and TRulsen used the Zakharov equation enhanced with the Krasitskii kernel (Krasitskii, 1994) to bring the Dysthe equation into Hamiltonian form, obtaining a new higher order nonlinear Schrödinger (HONLS) equation (Gramstad and Trulsen, 2011).

In order to examine rogue waves and downshifting in deep water we consider the HONLS equation of Gramstad and Trulsen with periodic boundary conditions, $u(x, t)=u(x+$ $L, t$ ), and add damping as follows: 


$$
\begin{aligned}
i u_{t} & +u_{x x}+2|u|^{2} u+i \Gamma u \\
& +i \epsilon\left(\frac{1}{2} u_{x x x}-8|u|^{2} u_{x}-2 u i(1+i \beta)\left[H\left(|u|^{2}\right)\right]_{x}\right)=0
\end{aligned}
$$

where $H(f)$ represents the Hilbert transform of $f$. Throughout this paper "HONLS equation" refers to (1) with $\Gamma=0$ and $\beta=0$. The Hamiltonian for the HONLS equation is given by

$$
\begin{aligned}
\mathcal{H} & =\int_{0}^{L}\left\{-i\left|u_{x}\right|^{2}+i|u|^{4}-\frac{\epsilon}{4}\left(u_{x} u_{x x}^{*}-u_{x}^{*} u_{x x}\right)\right. \\
& \left.+2 \epsilon|u|^{2}\left(u^{*} u_{x}-u u_{x}^{*}\right)+i \epsilon|u|^{2}\left[H\left(|u|^{2}\right)\right]_{x}\right\} d x .
\end{aligned}
$$

Uniform linear damping occurs for $\Gamma>0$ and $\beta=0$ and has been used extensively to study damping of wave trains with comparisons to physcial wave-tank experiments (Segur et al., 2005). Localized nonlinear damping of the mean flow occurs for $\epsilon, \beta>0$ and $\Gamma=0$ and it was introduced to investigate downshifting in deep water waves (Kato and Oikawa, 1995).

\subsubsection{Energy, energy flux, and the spectral center}

The mass or wave energy, $E$, and the momentum or total energy flux, $P$, are defined by

$E=\int_{0}^{L}|u|^{2} d x$, and $P=i \int_{0}^{L}\left(u^{*} u_{x}-u u_{x}^{*}\right) d x$.

To show damping occurs for positive $\beta$ or $\Gamma$, one finds that

$$
\frac{d E}{d t}=-2 \int_{0}^{L}|u|^{2}\left(\Gamma+2 \epsilon \beta\left[H\left(|u|^{2}\right)\right]_{x}\right) d x .
$$

Expanding $u$ and $|u|^{2}$ in Fourier series, $u=\sum_{k=-\infty}^{\infty} \hat{u}_{k} e^{i k x}$, $|u|^{2}=\sum_{k=0}^{\infty}\left(B_{k} e^{i k x}+B_{k}^{*} e^{-i k x}\right)$,

$\frac{d E}{d t}=-2 L\left[\Gamma \sum_{k=-\infty}^{\infty}\left|\hat{u}_{k}\right|^{2}+2 \epsilon \beta \sum_{k=1}^{\infty} k\left|B_{k}\right|^{2}\right]$.

Thus the total energy is conserved for the HONLS equation $(\Gamma=\beta=0)$ and is dissipated if either $\Gamma$ or $\beta$ is positive.

The total energy flux is related to the symmetry of the Fourier modes since $P$ can be written as

$$
P=-2 L \sum_{k=1}^{\infty} k\left(\left|\hat{u}_{-k}\right|^{2}-\left|\hat{u}_{k}\right|^{2}\right) \text {. }
$$

Since the evolution of the total energy flux is

$$
\frac{d P}{d t}=-2 i \int_{0}^{L}\left(u^{*} u_{x}-u u_{x}^{*}\right)\left(\Gamma+2 \epsilon \beta\left[H\left(|u|^{2}\right)\right]_{x}\right) d x
$$

the momentum is conserved by the HONLS equation. In contrast the momentum for the Dysthe equation exhibited small oscillations in time (Islas and Schober, 2010).
Using the Fourier spectrum of $u(x, t), \hat{u}_{k}$, two different choices of diagnostic frequencies can be used to measure downshifting. On the one hand, the dominant mode or spectral peak intuitively corresponds to the $k$ for which $\left|\hat{u}_{k}\right|$ achieves its maximum and is denoted as $k_{\text {peak }}$. On the other hand, Uchiyama and Kawahara defined the wave number for the spectral center or mean frequency of the spectrum as (Uchiyama and Kawahara, 1994):

$k_{\mathrm{m}}=-\frac{1}{2} \frac{P}{E}$.

The wave train is understood to experience a permanent frequency downshift when the spectral center $k_{\mathrm{m}}$ decreases monotonically in "time" or there is a permanent downshift of the spectral peak $k_{\text {peak }}$ (Trulsen and Dysthe, 1997a).

Given that the energy and momentum are conserved by the HONLS equation, the spectral center, which is given by their ratio, is also conserved. In the linearly damped model, $\Gamma>0, \beta=0$, from Eqs. (3) and (4) it follows that the energy and momentum have a simple time dependence:

$E(t)=E(0) \exp (-2 \Gamma t)$
$P(t)=P(0) \exp (-2 \Gamma t)$.

Thus the spectral center $k_{\mathrm{m}}$ is constant in time for the linearly damped HONLS equation and downshifting will not occur.

\subsubsection{Linear stability of the Stokes wave}

The HONLS equation admits a uniform wave train solution, the Stokes wave,

$u_{a}(x, t)=a e^{2 i a^{2} t}$.

Fourier analysis shows that this plane wave solution is unstable to sideband perturbations. When its amplitude $a$ is sufficiently large, for $0<\pi n / L<a$, the solution of the linearized NLS equation about $u_{a}$ has $M$ linearly unstable modes (UMs) $e^{i\left(\sigma_{n} t+2 \pi n x / L\right)}$ with growth rates $\sigma_{n}$ given by

$\sigma_{n}^{2}=\mu_{n}^{2}\left(\mu_{n}^{2}-4 a^{2}\right), \quad \mu_{n}=2 \pi n / L$,

where $M$ is the largest integer satisfying $0<M<a L / \pi$. We refer to this as the M-unstable mode regime.

\subsection{Integrable theory of the nonlinear Schrödinger equation}

In previous studies of sea states described by the JONSWAP power spectrum we showed a nonlinear spectral decomposition of the data provides relevant information on the likelihood of rogue waves. Here we briefly review the spectral theory of the nonlinear Schrödinger (NLS) equation,

$i u_{t}+u_{x x}+2|u|^{2} u=0$. 
The NLS equation is equivalent to the solvability condition of the AKNS system, the pair of first-order linear systems (Zakharov and Shabat, 1972):

$\mathcal{L}^{(x)} \phi=0, \quad \mathcal{L}^{(t)} \phi=0$,

for a vector-valued function $\phi$. The operator $\mathcal{L}^{(x)}$ is given by

$\mathcal{L}^{(x)}=\left(\begin{array}{cc}\partial_{x}+i \lambda & -u \\ u^{*} & \partial_{x}-i \lambda\end{array}\right)$

and depends on $x$ and $t$ through the potential $u$ and on the spectral parameter $\lambda$.

The nonlinear spectral decomposition of an NLS initial condition (or in general of an ensemble of JONSWAP initial data) is based on the inverse spectral theory of the NLS equation. For periodic boundary conditions $u(x+$ $L, t)=u(x, t)$, the Floquet spectrum associated with an NLS potential $u$ (i.e. the spectrum of the linear operator $\mathcal{L}^{(x)}$ at $u$ ) can be described in terms of the Floquet discriminant of $u$, defined as the trace of the transfer matrix of a fundamental matrix solution $\Phi$ of (11) over the interval $[0, L]$ (Ablowitz and Segur, 1981):

$\Delta(u ; \lambda)=\operatorname{Trace}\left(\Phi(x, t ; \lambda)^{-1} \Phi(x+L, t ; \lambda)\right)$.

Then, the Floquet spectrum is defined as the region

$\sigma(u)=\{\lambda \in \mathbb{C} \mid \Delta(u ; \lambda) \in \mathbb{R},-2 \leq \Delta \leq 2\}$.

Points of the continuous spectrum of $u$ are those for which the eigenvalues of the transfer matrix have unit modulus, and therefore $\Delta(u ; \lambda)$ is real and between 2 and -2 ; in particular, the real line is part of the continuous spectrum. Points of the L-periodic/antiperiodic discrete spectrum of $u$ are those for which the eigenvalues of the transfer matrix are \pm 1 , equivalently $\Delta(u ; \lambda)= \pm 2$. Points of the discrete spectrum which are embedded in a continuous band of spectrum are critical points for the Floquet discriminant (i.e., $\mathrm{d} \Delta / \mathrm{d} \lambda$ must vanish at such points).

Since the transfer matrix only changes by conjugation when we shift in $x$ or $t, \Delta$ is independent of those variables. An important consequence of this observation is that the Floquet discriminant is invariant under the NLS flow, and thus encodes an infinite family of constants of motion (parametrized by $\lambda$ ).

The continuous part of Floquet spectrum of a generic NLS potential consists of the real axis and of complex bands terminating in simple points $\lambda_{j}^{s}$ (at which $\Delta= \pm 2, \Delta^{\prime} \neq$ 0 ). The N-phase potentials are those characterized by a finite number of bands of continuous spectrum (or a finite number of simple points). Figure 1a shows the spectrum of a typical $\mathrm{N}$-phase potential: complex critical points (usually double points of the discrete spectrum for which $\Delta^{\prime}=0$ and $\Delta^{\prime \prime} \neq 0$ ), such as the one appearing in the figure, are in general associated with linear instabilities of $u$ and label its homoclinic orbits (Ercolani et al., 1990).

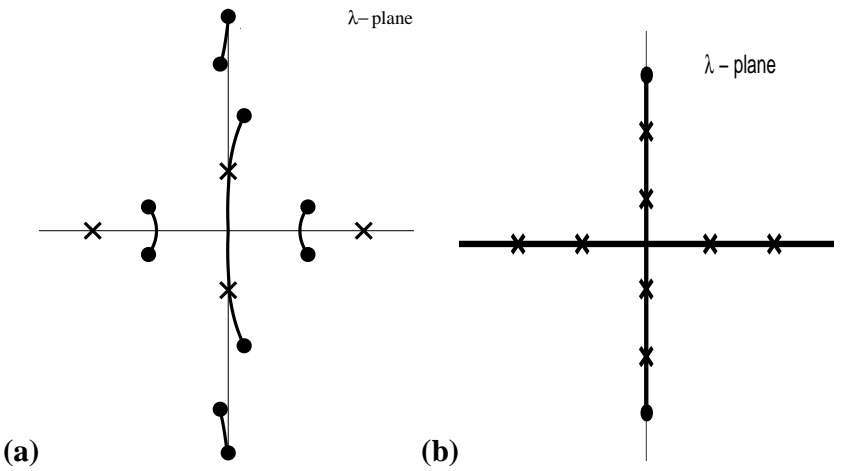

Fig. 1. Spectrum of (a) a generic unstable N-phase solution and (b) the plane wave solution. The simple periodic eigenvalues are labeled by circles and the double points by crosses.

As a concrete example consider the plane wave solution, $u_{a}(x, t)=a e^{2 i a^{2} t}$, whose Floquet discriminant is given by $\Delta(a, \lambda)=2 \cos \left(\sqrt{a^{2}+\lambda^{2}} L\right)$. The resulting Floquet spectrum (Fig. 1b) consists of the continuous bands $\mathbb{R} \bigcup[-i a, i a]$, and a discrete part containing the simple periodic/antiperiodic eigenvalues $\pm i a$, and the infinite sequence of double points

$\lambda_{j}^{2}=(j \pi / L)^{2}-a^{2}, \quad j \in \mathbb{Z}$.

The number of imaginary double points, obtained for $0 \leq j<$ $a L / \pi$, coincides with the number of unstable modes (Eq. 9) computed directly from the linearization. Each imaginary double point "labels" the associated unstable mode (Calini and Schober, 2002).

\section{Rogue waves in the NLS and HONLS equations}

\subsection{Homoclinic solutions of the NLS equation as models for rogue waves}

The NLS equation admits modulationally unstable periodic solutions with homoclinic orbits that can undergo large amplitude excursions away from their target solution. Such homoclinic orbits can be used to model rogue waves. An example is provided by the unstable plane wave solution $u_{a}(x, t)=a e^{2 i a^{2} t}$ where the number of unstable modes (UMs) is provided by Eq. (9). For each UM there is a corresponding homoclinic orbit. A global representation of the homoclinic orbits can be obtained by exponentiating the linear instabilities via Bäcklund transformations (Matveev and Salle, 1991; McLaughlin and Schober, 1992). Moreover, for NLS potentials with several UMs, iterated Bäcklund transformations will generate their entire stable and unstable manifolds, comprised of homoclinic orbits of increasing dimension up to the dimension of the invariant manifolds. Such higher-dimensional homoclinic orbits are also known as combination homoclinic orbits. 


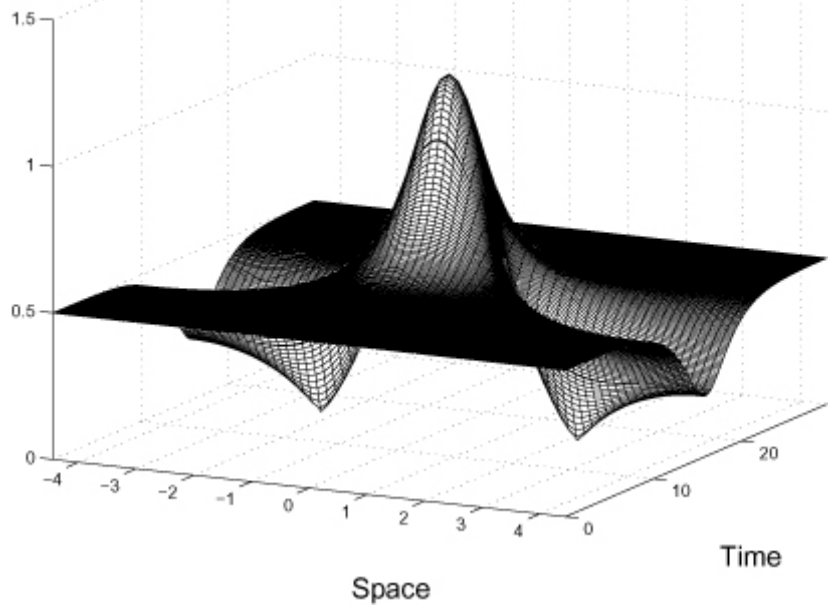

Fig. 2. Amplitude plot of a homoclinic orbit of the Stokes wave with $a=0.5, L=2 \sqrt{2} \pi$.

\subsubsection{One unstable mode rogue wave solutions}

A single (i.e. lowest dimensional) homoclinic orbit of the plane wave potential is given by

$u(x, t)=a e^{-2 i a^{2} t} \frac{1+2 \cos (p x) e^{\sigma_{n} t+2 i \phi+\rho}+A e^{2 \sigma_{n} t+4 i \phi+2 \rho}}{1+2 \cos (p x) e^{\sigma_{1} t+\rho}+A e^{2 \sigma_{n} t+2 \rho}}$

where $A=1 / \cos ^{2} \phi, \sigma_{n}= \pm p \sqrt{4 a^{2}-p^{2}}, \phi=\sin ^{-1}(p / 2 a)$, and $p=\mu_{n}=2 \pi n / L<a$ for some integer $n$. Each UM has an associated homoclinic orbit characterized by mode $p=$ $\mu_{n}$.

Figure 2 shows the space-time plot of the amplitude $|u(x, t)|$ of a homoclinic orbit with one UM, for $a=0.5$, $L=2 \sqrt{2} \pi$ and $p=2 \pi / L$. As $t \rightarrow \pm \infty$, solution (13) limits to the plane wave potential; in fact, the plane wave behavior dominates the dynamics of the homoclinic solution for most of its lifetime. As $t$ approaches $t_{0}=0$, nonlinear focusing occurs due to the BF instability and the solution rises to a maximum height of $2.4 a$. Thus, the homoclinic solution with one UM can be regarded as the simplest model of rogue wave.

An almost equally dramatic wave trough occurs close to the crest of the rogue wave as a result of wave compression due to wave dislocation. The amplitude amplification factor is given by

$A f=\frac{\max _{x \in[0, L], t \in \mathbf{R}}|u(x, t)|}{\lim _{t \rightarrow \pm \infty}|u(x, t)|} \approx 2.4$

www.nat-hazards-earth-syst-sci.net/11/383/2011/

\subsubsection{Phase modulated rogue waves}

As the number of UMs increases, the space-time structure of the homoclinic solutions becomes more complex. When two or more UMs are present the initial wave train can be phase modulated to produce additional focusing.

The family of homoclinic orbits of the plane wave potential with two UMs is given by an expression of the form

$u(x, t)=a e^{2 i a^{2} t} \frac{g(x, t)}{f(x, t)}$,

where the expression for $f(x, t)$ and $g(x, t)$ depend on the two spatial modes $\cos (2 n \pi x / L), \cos (2 m \pi x / L)$, and on temporal exponential factors $\exp \left(\sigma_{n} t+\rho_{n}\right), \exp \left(\sigma_{m} t+\rho_{m}\right)$, with growth rates $\sigma_{l}=\mu_{l} \sqrt{\mu_{l}^{2}-4 a^{2}}, \mu_{l}=2 \pi l / L$. (The complete formulas can be found in Calini et al., 1996; Calini and Schober, 2002.)

As in the one-UM case, this combination homoclinic orbit decays to the plane wave potential as $t \rightarrow \pm \infty$, and the associated rogue wave remains hidden beneath the background plane wave for most of its lifetime. The temporal separation of the two spatial modes depends upon a parameter $\rho$ related to the difference $\rho_{n}-\rho_{m}$ in the temporal phases (Calini et al., 1996; Calini and Schober, 2002).

In turn, $\rho$ affects the amplitude amplification factor. Figure $3 \mathrm{a}-\mathrm{b}$ shows the combination homoclinic orbit (15) obtained with all parameters set equal except for $\rho$. In Fig. 3a, $\rho=.1$, the modes are well separated, and the amplitude amplification factor is roughly three. In Fig. 3b, the value of $\rho$ is approximately -0.65 , corresponding to the two UMs being simultaneously excited or coalesced. For this value of $\rho$ the amplitude amplification factor is maximal and the rogue wave rises to a height of 4.1 times the height of the carrier wave. The surface plot of $|u(x, t)|$ has been rotated in Fig. $3 b$ to facilitate comparison with Fig. 4a.

Figure 3 a shows focusing due to only weak amplitude modulation of the initial wave train; the growth in amplitude beginning at $t \approx 10$ and at $t \approx 25$ is due to the BF instability. However, in Fig. $3 \mathrm{~b}$ focusing due to both amplitude and phase modulation occurs. The amplitude growth at $t \approx 10$ is due to the BF instability, while the additional very rapid focusing at $t \approx 18.4$ is due to the phase modulation. In general it is possible to select the phases in a combination homoclinic orbit with $N$ spatial modes so that any number $n$ $(2 \leq n \leq N)$ of modes coalesce at some fixed time.

\subsection{Rogue waves in the higher order NLS equation}

In this section we examine the robustness of homoclinic solutions of the NLS equation, as well as frequency downshifting, when higher order terms are included in the wave dynamics. The HONLS equation (1) is solved numerically using a smoothed exponential time differencing integrator. The details of the method are provided in Sect. 4 


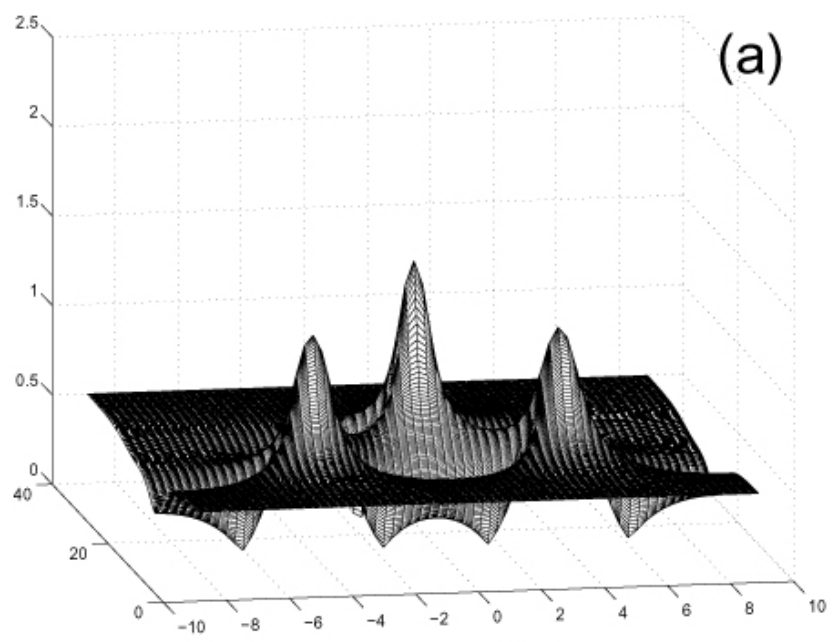

(b)

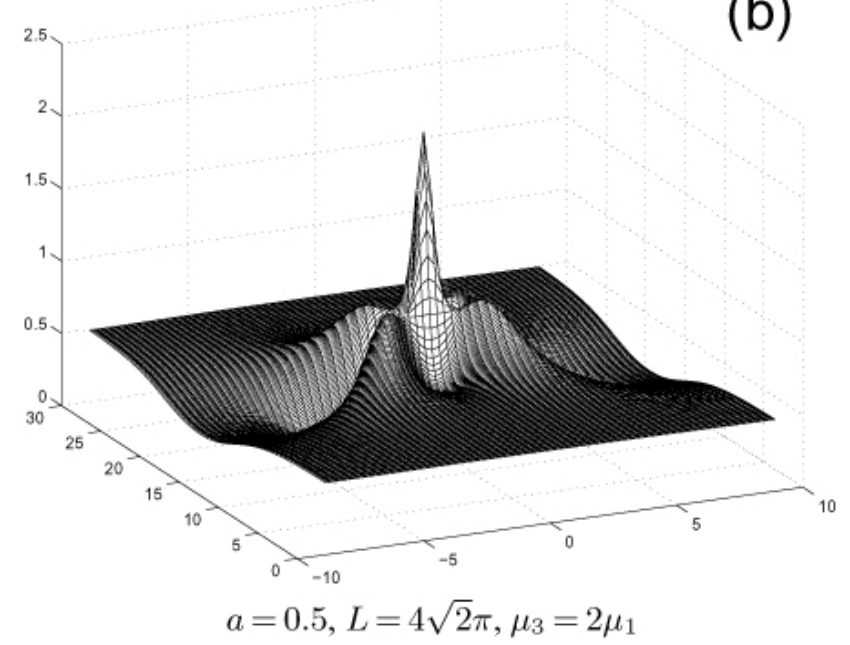

Fig. 3. Amplitude plots of the combination homoclinic orbit: (a) the phases are selected to produce well separated spatial modes; (b) shows a coalesced homoclinic orbit.

and in references (Cox and Matthews, 2002; Khaliq et al., 2009). As expected, we find permanent downshifting does not occur in the HONLS equation. We find that the chaotic background increases the likelihood and amplitude of rogue waves as compared to predictions obtained with the NLS equation.

We choose initial data that are small perturbations of the unstable plane wave potential,

$u(x, 0)=a(1+0.1 \cos \mu x)$,

where the amplitude $a$ is varied to be in the two or three unstable mode regime with $\mu=2 \pi / L$ and $L=4 \sqrt{2} \pi$. Figure $4 \mathrm{a}$ illustrates a prominent rogue wave solution of Eq. (1), $\epsilon=0.05, \beta=\Gamma=0$ for initial data (16) in the twoUM regime with $a=0.5,456<t<461$. The solution rapidly becomes chaotic (around $t=27$ ) with rogue waves emerging intermittently afterwards. For example, at $t \approx$
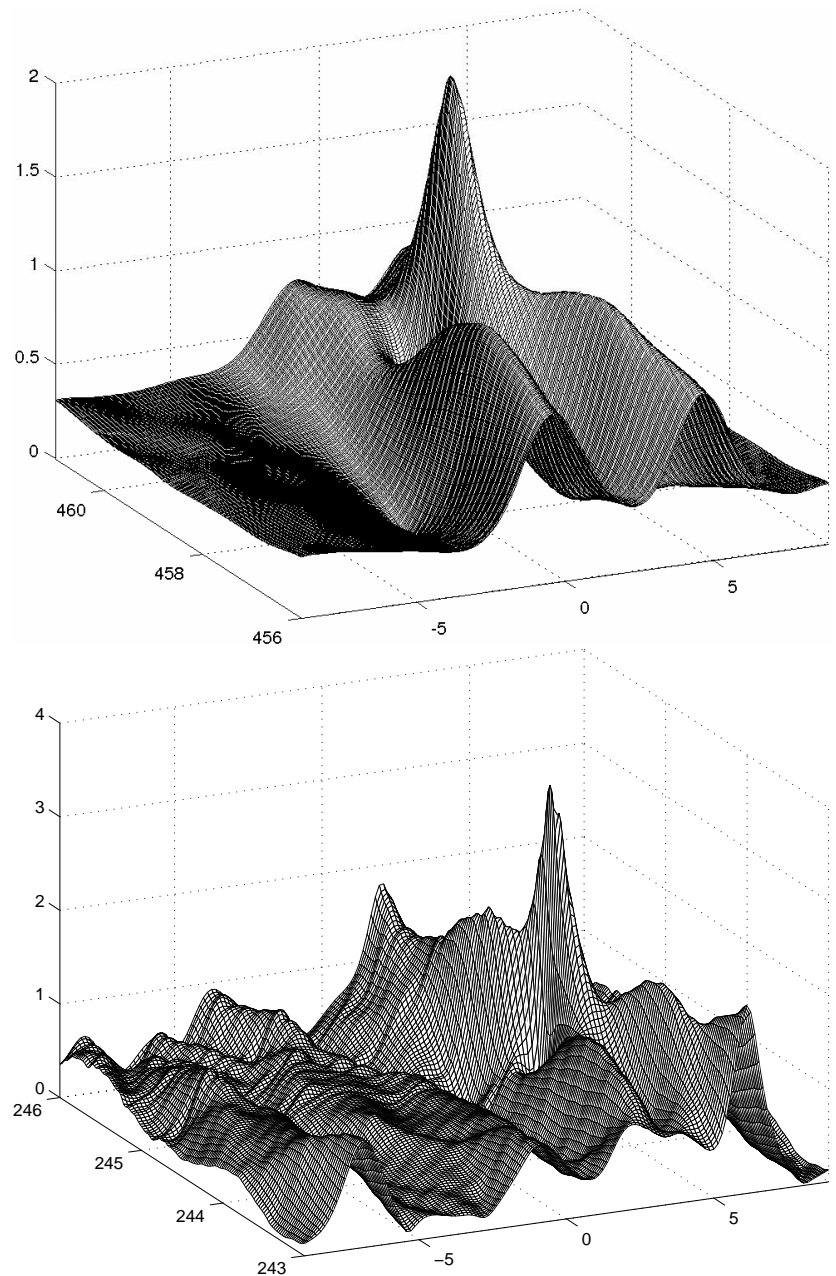

Fig. 4. Rogue waves solutions for the HONLS equation $(\epsilon=.05$, $\Gamma=\beta=0$ ) when (a) two and (b) three unstable modes are present.

459 a rogue wave rises with a maximum wave amplitude $U_{\max } \approx 2.15$. Comparing Fig. $4 \mathrm{a}$ with Fig. $3 \mathrm{~b}$ one finds that the structure of this rogue wave is similar to that of the combination homoclinic solution (15) with coalesced spatial modes obtained when $\rho=-0.65$, although the wave amplification factor is slightly smaller. The symmetry breaking effects of the higher order terms in the HONLS equation prevent a complete spatial coalescence of the nonlinear modes.

Numerical simulations of the HONLS equation in the three-UM regime, e.g. initial condition (16) with $a=0.7$, show a similar phenomenon: after the onset of chaotic dynamics, rogue waves rise intermittently above the chaotic background (see Fig. $4 \mathrm{~b}$ ). At $t \approx 245$ a rogue wave develops, $U_{\max } \approx 3.59$, which is close to the coalesced homoclinic solution of the NLS equation in the three-UM regime.

Extensive numerical experiments were performed for the HONLS equation in the two- and three-UM regime, varying 
the perturbation strength $\epsilon$, the amplitude $a$ and adding in some random phases $\phi_{i}$ 's in the initial data. In all cases, the coalesced homoclinic NLS solution emerges generically as a structurally stable feature of the perturbed dynamics.

The occurence of rogue wave in the HONLS equation is distinct from their occurence in the NLS or the damped HONLS equations. In the HONLS equation, rogue waves will occur intermittently throughout very long time series. In the NLS equation they occur only once or twice, depnding on whether the modes are coalesced or not. In the damped HONLS, they occur irregularly over a short time period but eventually are damped out.

The chaotic regime produces additional focusing by effectively selecting optimal phase modulations and the chaotic dynamics singles out the maximally coalesced homoclinic solutions of the unperturbed NLS equation as physically observable rogue waves. Moreover, the likelihood of larger amplitude waves (see e.g. Fig. 4) increases for the HONLS equation, as substantiated by the diagnostics developed in Sect. 5, correlating wave strengths in the NLS and HONLS models to proximity to homoclinic data. Thus, for initial data in the neighborhood of an unstable plane wave the underlying chaotic dynamics of the HONLS equation favors the occurrence of large amplitude rogue waves, as compared to predictions obtained from the NLS equation.

To investigate downshifting we varied the perturbation strength $\epsilon$ in the HONLS equation and the parameters in the initial data in the two- and three-UM regime. Typical results obtained with the HONLS equation (1) are shown in Fig. 5 for initial data

$$
\begin{aligned}
u(x, 0) & =0.7(1+0.1(\cos \mu x+0.2(\cos 2 \mu(x-L / 4) \\
& +0.38 \cos 3 \mu(x-L / 3))))
\end{aligned}
$$

with $L=4 \sqrt{2} \pi$ and $\epsilon=0.05$.

Figure 5a shows the time evolution of the strength of $u(x, t)$,

$S(t)=\frac{U_{\max }(t)}{H_{\mathrm{S}}(t)}$,

where $U_{\max }(t)=\max _{x \in[0, L]}|u(x, t)|$ and $H_{\mathrm{S}}(t)$ is the significant wave height, defined as 4 times the standard deviation of the surface elevation. Rogue waves occur throughout the time series whenever the strength exceeds the threshold criteria of 2.2. Figure $5 \mathrm{~b}$ shows the time evolution of the main Fourier modes $\left|A_{k}(t)\right|$ for $k=0, \pm 1, \ldots, \pm 4$. During each of the modulation stages the zeroth mode loses energy as the upper and lower higher harmonics become excited. For $0<t<200$ the total energy and the total energy flux are constant. This allows the energy to flow back to the zeroth mode keeping the spectral center $k_{m}$ constant (Fig. 5c). The plot of $k_{\text {peak }}$ (Fig. 5d) confirms that permanent frequency downshifting does not occur. As expected, even when steeper waves are obtained, permanent frequency downshifting does not occur in the HONLS numerical experiments, indicating the necessity of a dissipative or nonconservative process.
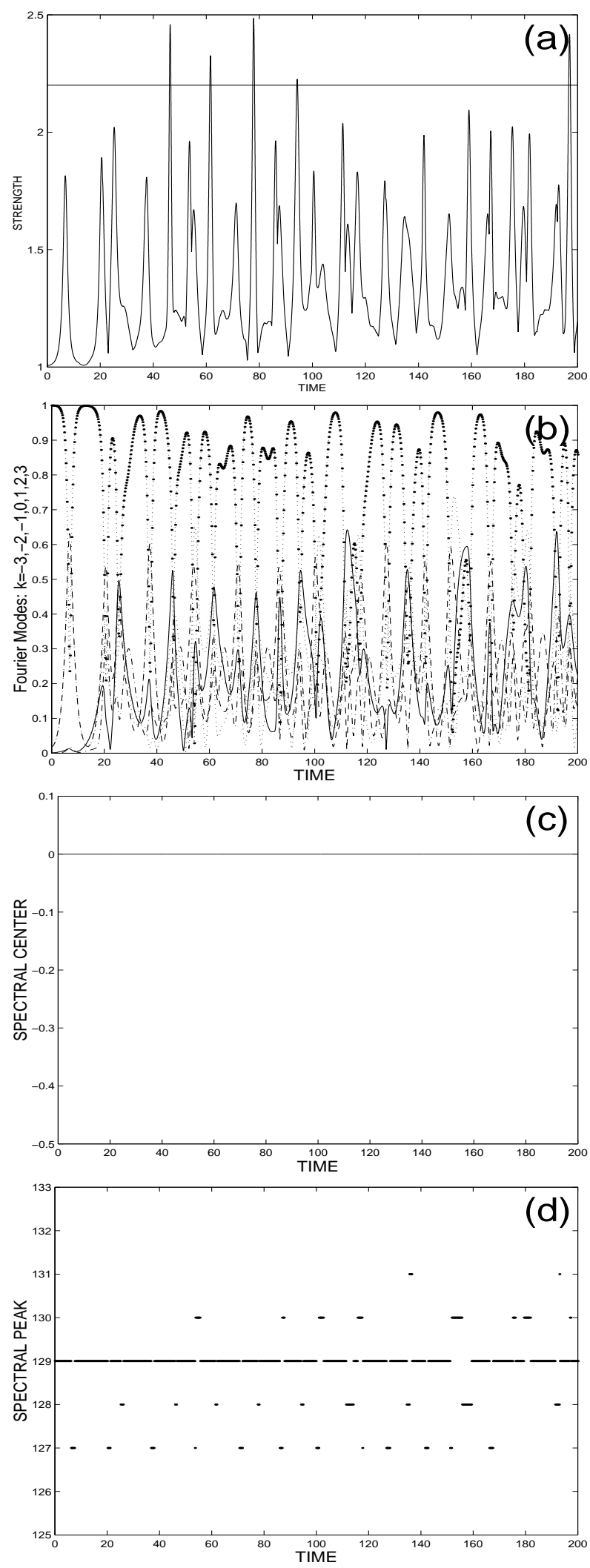

Fig. 5. The HONLS equation, $\Gamma=0, \epsilon=0.05, \beta=0$ : time evolution of (a) the strength $S(t)$, (b) the main Fourier mode, (c) the spectral center $k_{\mathrm{m}}$, and (d) the spectral peak $k_{\text {peak }}$. 


\section{Rogue waves and downshifting in the presence of damping}

In the last section we saw that an $\epsilon$-neighborhood of the unstable plane wave with two or more UMS is effectively a rogue wave regime for the HONLS equation as the probability of obtaining a rogue wave is high. In the numerical experiments we choose initial data that are small perturbations of an unstable plane wave to study the effects of linear and nonlinear damping on the development of rogue waves and downshifting. We consider the damped HONLS equation in non-dimensional form with the damping terms on the same order or smaller than the higher order NLS terms. Consequently we vary the damping coefficients to be $0<\epsilon \beta, \Gamma<O(\epsilon)$, where $\epsilon$ is the coefficient of the higher order NLS terms.

In the numerical experiments Eq. (1) is solved using a smoothing fourth-order exponential time differencing integrator (Cox and Matthews, 2002; Khaliq et al., 2009) that avoids inaccuracies when inverting matrix polynomials by using Pade approximations. We use $N=256$ Fourier modes in space and a fourth-order Runge-Kutta discretization in time $\left(\Delta t=10^{-3}\right)$. The high frequency modes were eliminated at every time step to avoid aliasing by setting $\hat{u}_{k}=$ 0 for $|k|>120$. Eliminating the high frequencies does not have a significant effect on the exchange of energy between the dominant low wave number modes and thus does not impact our results related to frequency downshifting and rogue waves.

This temporal and spatial resolution allows for the three integral invariants of the conservative HONLS equation, $E$, $P$, and $\mathcal{H}$, to be conserved with an accuracy of $\mathcal{O}\left(10^{-8}\right)$, $\mathcal{O}\left(10^{-2}\right), \mathcal{O}\left(10^{-2}\right)$, respectively. The nonlinear mode content of the data is numerically computed using the direct spectral transform described above, i.e. the system of ODEs (11) is numerically solved to obtain the discriminant $\Delta$. The zeros of $\Delta \pm 2$ are then determined with a root solver based on Muller's method (Ercolani et al., 1990). The spectrum is computed with an accuracy of $\mathcal{O}\left(10^{-6}\right)$, whereas the spectral quantities we are interested in range from $\mathcal{O}\left(10^{-3}\right)$ to $\mathcal{O}\left(10^{-1}\right)$.

\subsection{The HONLS equation with linear damping}

We begin by examining the evolution of damped uniform wavetrains with initially one to three pairs of sidebands excited. It is important to note in comparisons of the damped and undamped dynamics that atypical cases may arise. Figure $6 \mathrm{a}-\mathrm{b}$ shows the strength of the wavetrain (solid line) and $U_{\max }$ (dashed line) for the undamped and linearly damped $(\Gamma=0.005)$ HONLS equation $(\epsilon=0.05)$, respectively, for initial data (16) in the two-UM regime: $a=0.5, \mu=2 \pi / L$ and $L=4 \sqrt{2} \pi$. Contrary to what one might expect, Fig. $6 \mathrm{~b}$ shows a rogue wave occuring at $t \approx 23$ in the damped case with an enhanced strength $\approx 2.8$ that
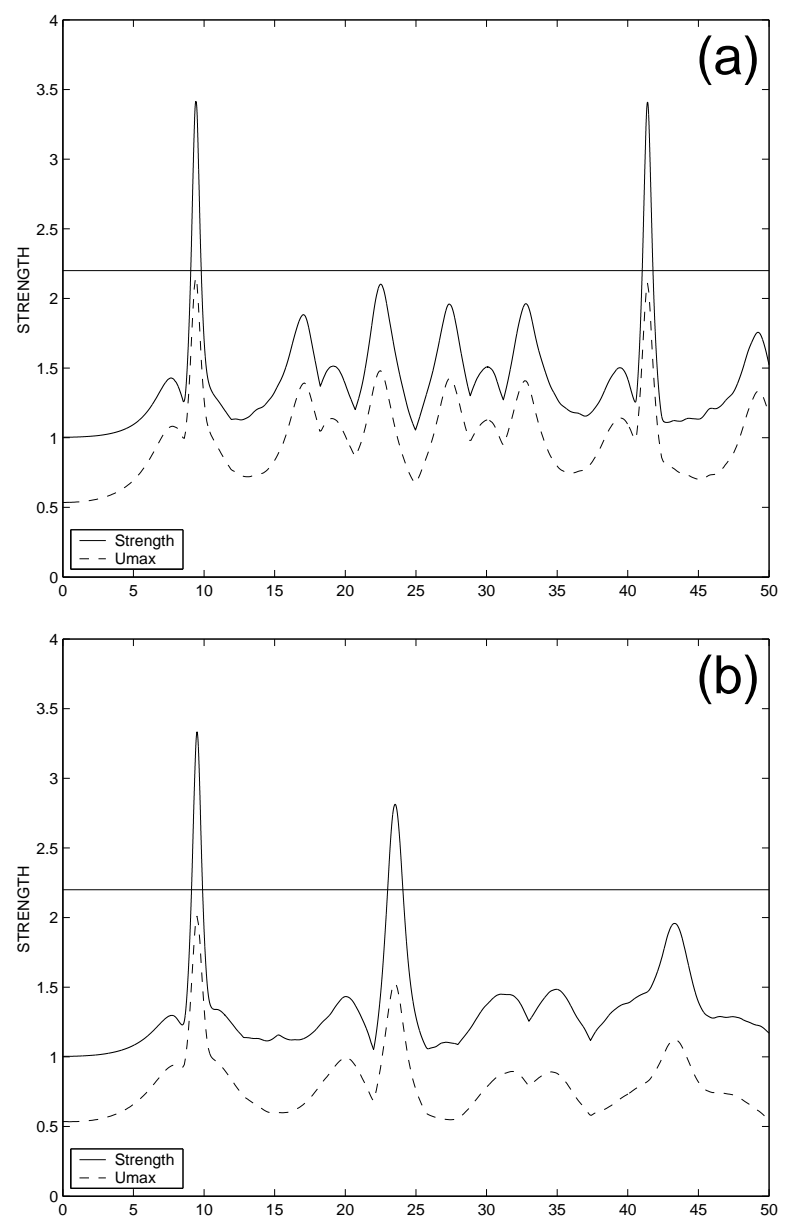

Fig. 6. Strength $S(t)$ (solid line) and $U_{\max }$ (dashed line) for (a) HONLS equation $\epsilon=0.05$; (b) HONLS equation with linear damping, $\epsilon=0.05, \Gamma=0.006$, for IC $u_{0}=0.5(1+0.1 \cos \mu x)$.

was not present in the undamped evolution (Fig. 6a). This typically occurs for small values of $\Gamma$ or $\beta$ where the smaller growth rates of the individual modes due to damping can be offset by a coalescence of the modes due to changes in their focusing times.

We examined the HONLS equation with linear damping for $0.005 \leq \Gamma \leq 0.05$. Although individual simulations may yield larger amplification factors in the damped regime, we find that on average, in the presence of linear damping (see Sect. 5.2 for confirmation with the nonlinear spectral diagnostics), the strength is smaller and fewer rogue waves occur. Figure 7 shows the results obtained with Eq. (1) for $\epsilon=0.05, \Gamma=0.01, \beta=0$ for initial condition (17) for $0<t<200$. In the linearly damped evolution, Fig. $7 \mathrm{a}$, the last rogue wave occurs at $t \approx 10$; in contrast, in Fig. 5a rogue waves occur throughout the unperturbed HONLS time series. The total energy exponentially decays (Eq. 6) and the Fourier modes are uniformly damped. The total energy flux is constant, keeping the spectral center $k_{m}$ zero. Permanent downshifting does not occur for the linearly damped HONLS equation. 

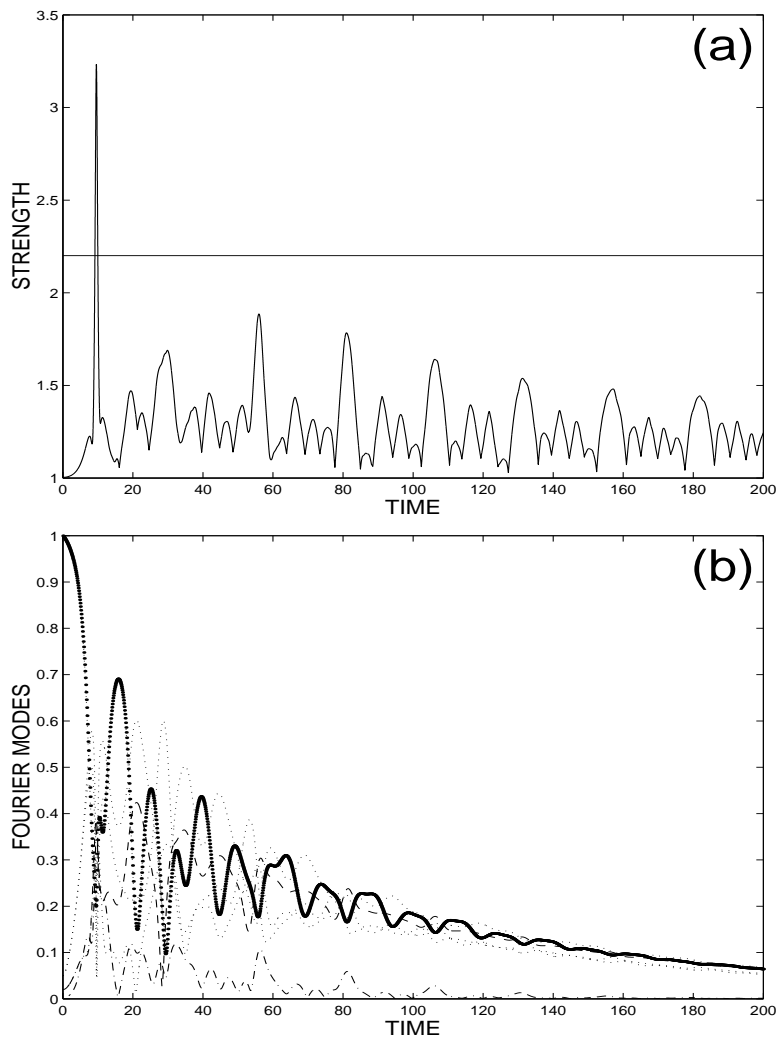

Fig. 7. The HONLS eq. with linear damping: $\Gamma=0.01, \epsilon=0.05$, $\beta=0$. The time evolution of (a) the strength $S(t)$ (b) the main Fourier modes, $0<t<200$.

\subsection{The HONLS Equation with nonlinear damping}

The perturbation of the Hilbert tranform term in Eq. (1), $\beta u\left[H\left(|u|^{2}\right)\right]_{x}$. is referred to as the " $\beta$-term" and models nonlinear damping of the mean flow. Figure 8 shows the spatial distribution of $\left|u\left(x, t^{*}\right)\right|$ (solid line) and of the $\beta$-term (dashed line) of the nonlinearly damped HONLS equation for $\epsilon=0.05, \beta=0.1, \Gamma=0$, for initial condition (16). At $t=22.7$ the wavetrain is strongly modulated and we find that the $\beta$-term is significant only near the maximum crest of the envelope. In general, steeper waves result in stronger damping and the effects of the nonlinear $\beta$-term are concentrated in space-time when the wavetrain is strongly modulated.

To illustrate the effects of nonlinear damping on downshifting we consider the solution of Eq. (1) with $\epsilon=$ $0.05, \beta=0.5$ and $\Gamma=0$, for initial data (17), $0<t<50$. Figure 9a-b shows the surface amplitude $|u(x, t)|$ and the time evolution of the strength, respectively. Two rogue waves occur early in the time series, $t<10$, before damping has a chance to significantly alter the growth rate of the modes and prevent further rogue waves from forming. Comparing Fig. $9 \mathrm{~b}$ and $9 \mathrm{c}$, the onset of downshifting occurs when the first rogue wave appears at approximately $t \approx 6$ when the $\beta$ -

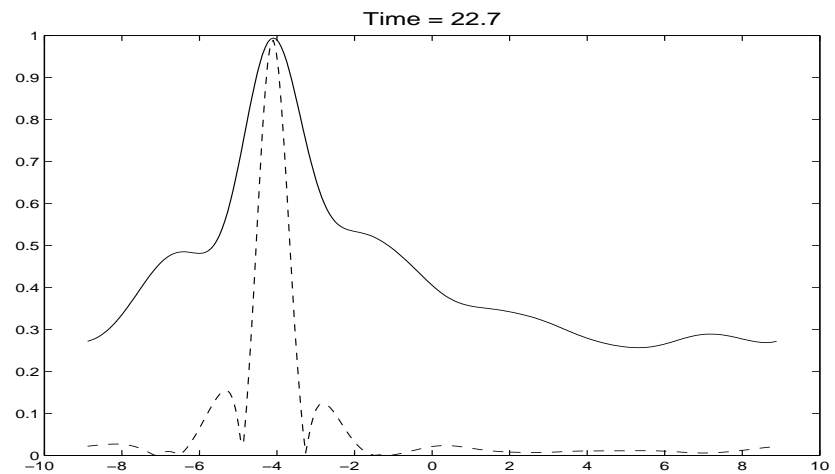

Fig. 8. Spatial distribution of $\left|u\left(x, t^{*}\right)\right|$ (solid line) and of the $\beta$-term (dashed line) at $t^{*}=22.7$ for initial condition (16), for $\epsilon=0.05$, $\beta=0.1, \Gamma=0$.

term is large. This produces an abrupt rapid decay in the energy (Fig. 9d) and growth in the flux (Fig. 9e), resulting in a rapid shift in the spectral center $k_{\mathrm{m}}$ (Fig. 9f). The downshifting becomes irreversible after $t \approx 17$ when the wavetrain is demodulated and the lower modes $k=-1,-4$ become dominant. In the demodulation stage the $\beta$-term is small so that the energy and flux change at a much slower rate. Thus $k_{\mathrm{m}}$ remains negative and does not upshift back.

Permanent downshifting is obtained for all values of $\beta$. As an example consider the solution of Eq. (1) with $\epsilon=0.05$, $\beta=0.04, \Gamma=0$ for initial data (17), $0<t<200$. For small $\beta$ there is a slow gradual decay in the total energy and flux rather than the abrupt rapid decay obtained for larger $\beta$. As a result the last rogue wave appears at $t \approx 51$ (Fig. 10a), much later than for $\beta=0.5$. The plot of the evolution of the main Fourier modes (Fig. 10b) shows permanent downshifting occurs at $t \approx 65$. Figure 11 , which shows the evolution of $k_{\text {peak }}$ for the nonlinearly damped HONLS equation $(\epsilon=0.05$, $\Gamma=0$ ) with $\beta=0.04$ or $\beta=0.5$, provides an alternate view of permanent downshifting for $\beta \neq 0$ and its occurence at later times for smaller values of $\beta$.

When both linear and nonlinear damping are present and the nonlinear damping is dominant, the mechanism for permanent downshifting is the same. Figure 12 shows the solution of Eq. 1 with $\epsilon=0.05, \beta=0.5, \Gamma=0.005$ for initial data (17), $0<t<50$. Comparing to Fig. 9, the rogue waves occur at approximately the same time with slightly smaller amplitudes. An abrupt rapid decay in the energy and growth in the flux occurs as before leading to a rapid shift in the spectral center $k_{\mathrm{m}}$.

\section{Characterization of the nonlinearly damped evolution}

To characterize the effects of nonlinear damping on rogue waves we fix $\epsilon=0.05$ and $\Gamma=0$ in Eq. (1) and vary the nonlinear damping coefficient, $0<\beta<0.75$, as well as the 
(a)
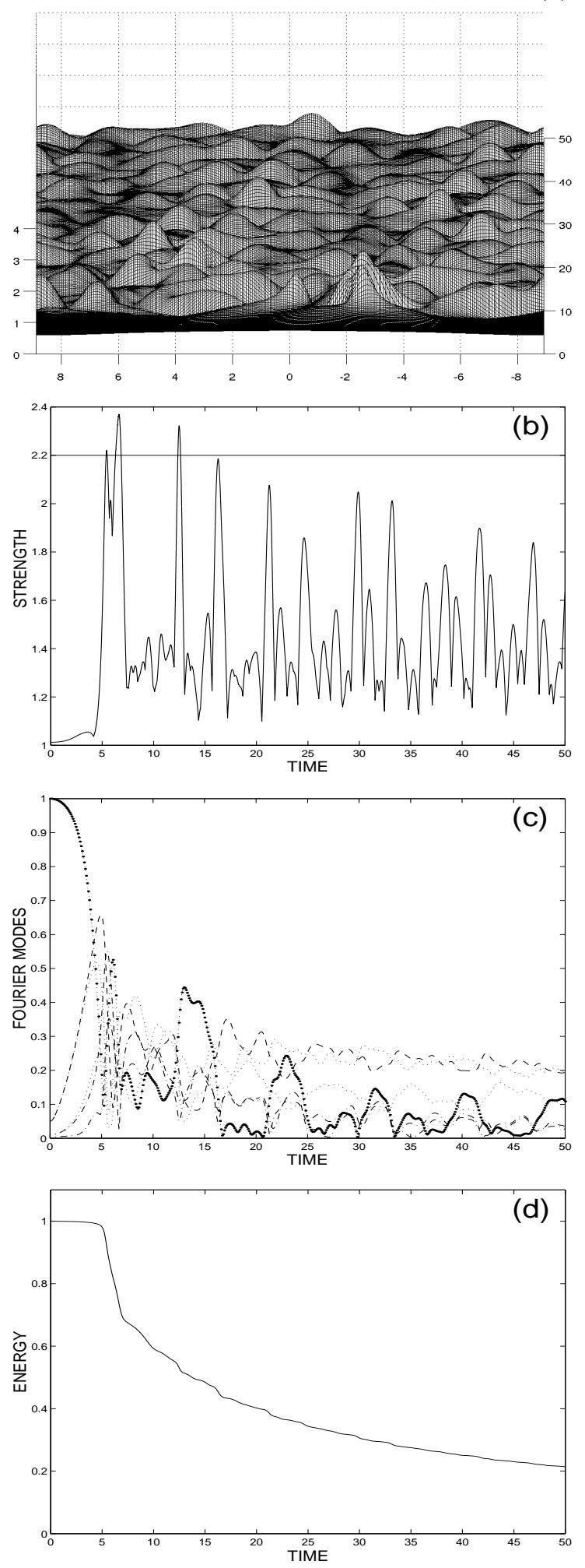

Fig. 9. Nonlinearly damped HONLS equation $\epsilon=0.05, \beta=0.5$, $\Gamma=0$ : (a) surface amplitude $|u(x, t)|$, and the time evolution of (b) the strength $S(t)$, (c) the main Fourier modes, (d) the total energy, for initial data (17), the time evolution of (e) the total energy flux, and (f) the spectral center $k_{\mathrm{m}}$ for initial data (17).
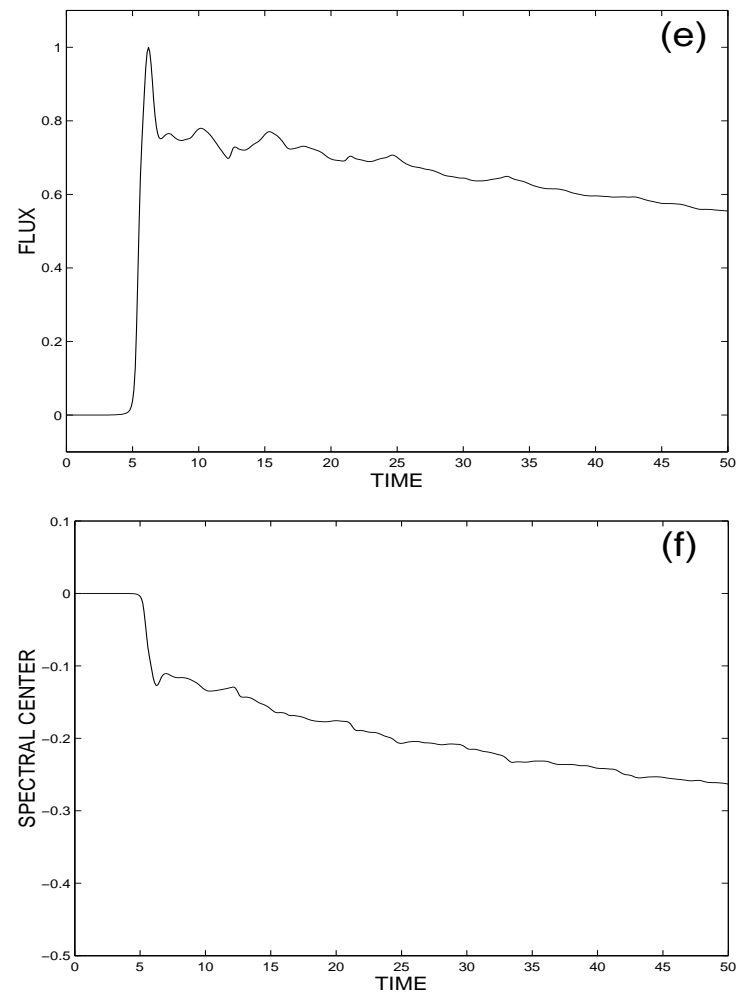

Fig. 9. Continued.

amplitude of the initial data in the three-UM regime:

$u(x, 0)=a(1+0.01 \cos \mu x)$,

with $0.57<a<0.67, \mu=2 \pi / L, L=4 \sqrt{2} \pi$. Figure 13 shows, as a function of $\beta$ : (a) the final value of the spectral center $k_{\mathrm{m}}$, (b) the maximum strength $\max _{t \in[0,200]} S(t)$, (c) the number of rogue waves obtained for $0<t<200$, and (d) the time of the last rogue wave. The solid curve represents the averages over the initial data. In the numerical experiments we observe the following:

1. The spectral center $k_{\mathrm{m}}(t)$ is a decreasing function of $t$ for all $\beta>0$. Figure 13a is not showing the time evolution of $k_{\mathrm{m}}$, (see e.g. Fig. 9f for a typical example), but rather the final value of $k_{\mathrm{m}}$ at $t=200$, where smaller values mean a downshift to a lower mode. Thus, permanent downshifting is observed for all $\beta>0$ with the onset occuring rapidly for larger values of $\beta$.

2. Figure 13b-c shows that for small values of $\beta$ the average maximum strength in time and average number of rogue waves exhibit irregular behavior. We may obtain more or larger rogue waves in the damped regime than without damping for this time frame due to changes in the focusing time and coalescence of the modes. However for large $\beta$, the maximum strength in time and number of rogue waves are, in general, decreasing. 

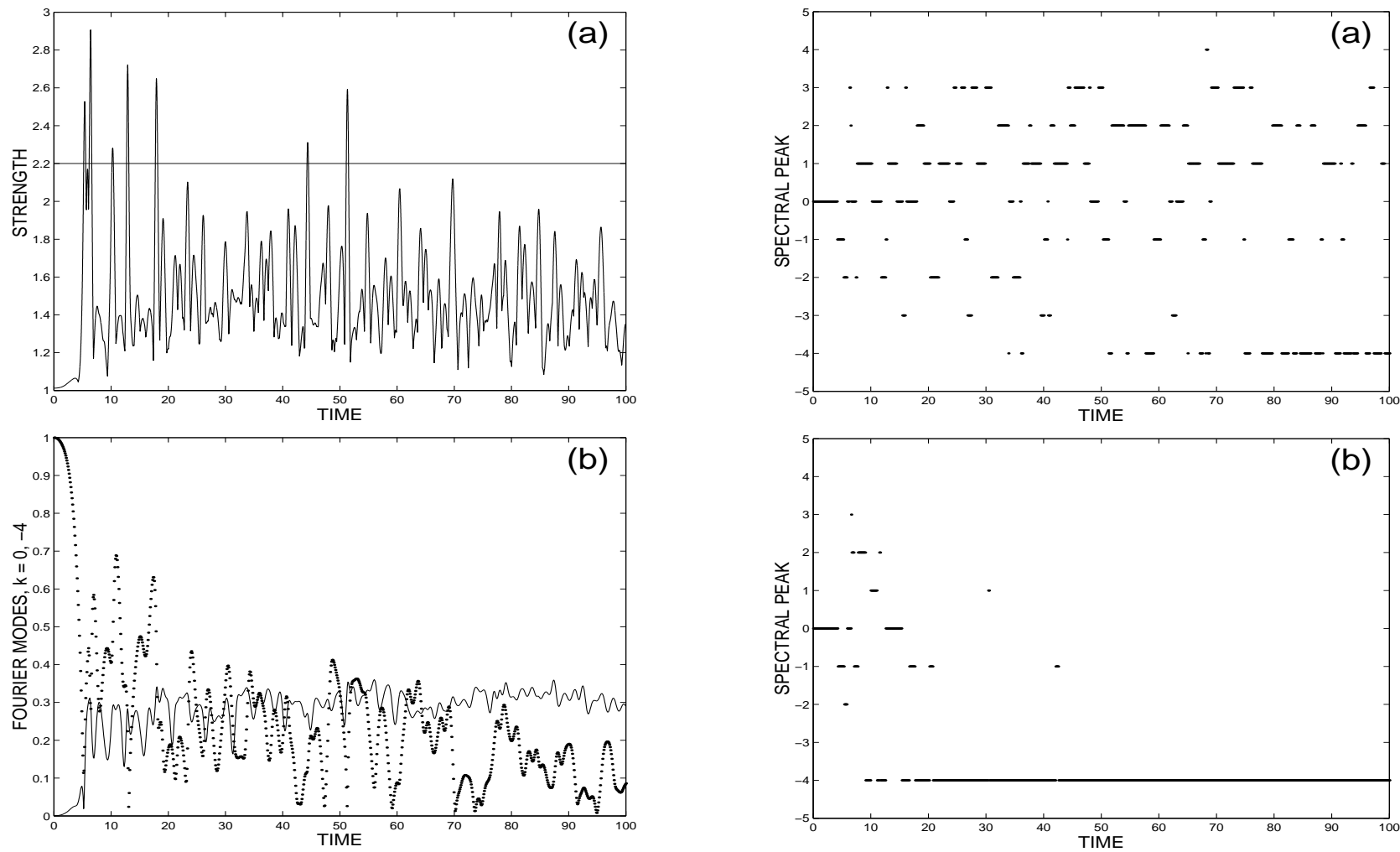

Fig. 10. The HONLS equation with nonlinear damping $\epsilon=0.05$, $\beta=0.04, \Gamma=0$ : time evolution of (a) the strength $S(t)$ and (b) the main Fourier modes for initial data (17).

3. For all values of the initial amplitude, the first rogue wave is only slightly delayed $(t \approx 6)$ for all $\beta$ considered (not shown). The time of the last rogue wave, $T_{1}$, may slightly increase for small $\beta$. As $\beta$ increases, $T_{1}$ significantly decreases, indicating fewer rogue waves occur. For $\beta>\beta_{*}$ only the initial pair of rogue waves develop (Fig. 13d) as large $\beta$ values trigger sufficient nonlinear damping that inhibit further rogue wave formation.

\section{Relation of downshifting and rogue waves}

It is significant in Figs. $9 \mathrm{~b}-\mathrm{c}$ and $10 \mathrm{a}-\mathrm{b}$ that permanent downshifting occurs after the last rogue wave in the time series. In other words, rogue waves do not occur after the downshifting becomes irreversible in these examples. To generalize this result we analyze the previous series of numerical experiments using initial data (19) with $0.57<$ $a<0.67$ to determine (1) the time of the last rogue wave and (2) the last time $k_{\text {peak }}$ is equal to $k_{0}$, indicating permanent downshifting, as the perturbation strength $\beta$ is varied.

Figure 14 compares the time at which downshifting becomes irreversible (labeled by an $\mathrm{x}$ ) with the time at which the last rogue wave occurs (labeled by a box) for the HONLS equation with only nonlinear damping, $\epsilon=0.05, \Gamma=0$ and

Fig. 11. The evolution of $k_{\text {peak }}$ for the nonlinearly damped HONLS equation with $\epsilon=0.05, \Gamma=0$ and (a) $\beta=0.04$ or (b) $\beta=.5$ for initial data (17).

$0<\beta<0.75$. Figure 14a shows the comparison for initial data (19) with $a=0.63$ : we observe that for all values of $\beta$ rogue waves do not occur after the downshifting becomes irreversible. Further, rogue waves do not develop after permanent downshifting occurs for any other pair of parameter values $(a, \beta)$ considered in the experiments. This is summarized in Fig. 14b which compares, for $0<\beta<$ 0.75 , the average time of the last rogue wave (box) with the average time at which downshifting is irreversible (x), where the averages are over the six simulations with initial data amplitude $0.57<a<0.67$. These results imply permanent downshifting serves as an indicator that the cumulative effects of damping are sufficient to prevent the further development of rogue waves.

Finally we consider the case when both linear and nonlinear damping are present and the nonlinear damping is dominant to allow permanent downshifting to occur. Figure 15 compares the times for permanent downshifting and the last rogue wave for the damped HONLS equation with $\epsilon=0.05, \Gamma=0.005$ and $0<\beta<0.75$. The same relation between downshifting and the last rogue wave are observed to hold, i.e. rogue waves do not occur after the downshifting is permanent. However, due to the additional linear damping there is a longer time lag between the last rogue wave and permanent downshifting. 
A. Islas and C. M. Schober: Rogue waves and downshifting
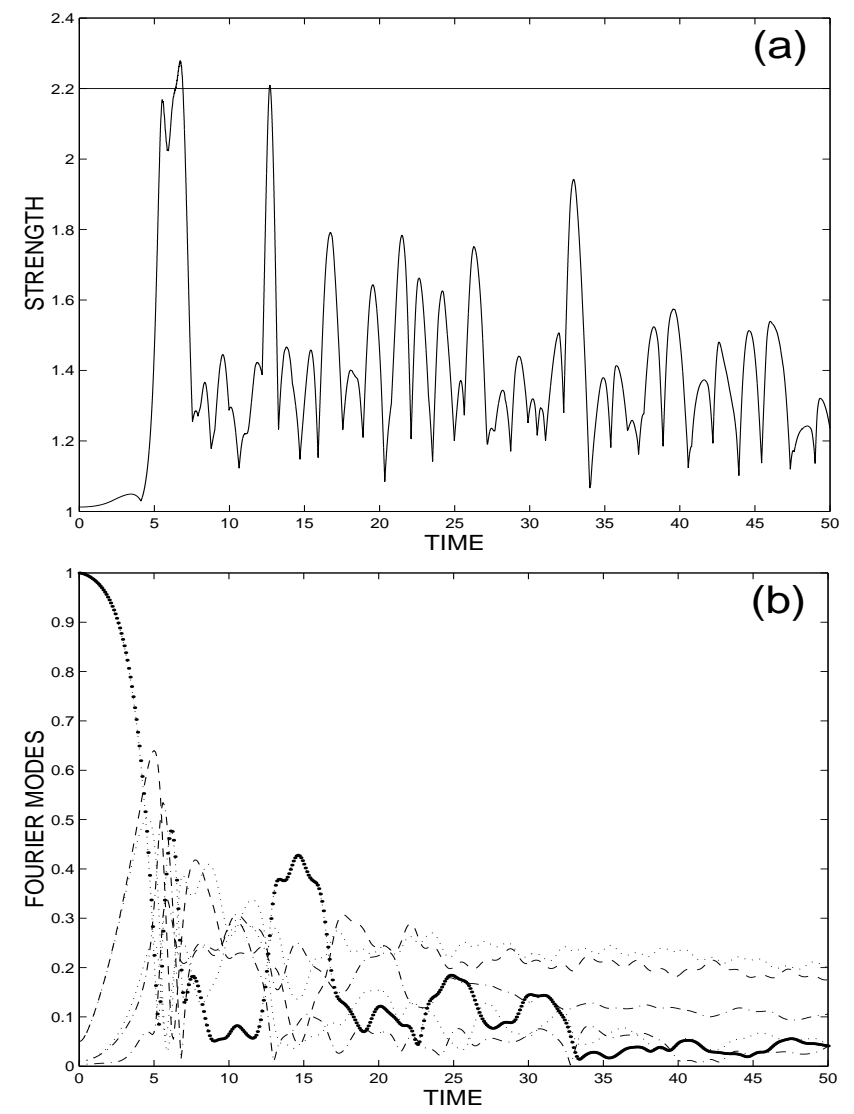

Fig. 12. Nonlinearly damped HONLS equation $\epsilon=0.05, \beta=0.5$, $\Gamma=0.005$ : the time evolution of (a) the strength $S(t)$, (b) the main Fourier modes, for initial data (17).

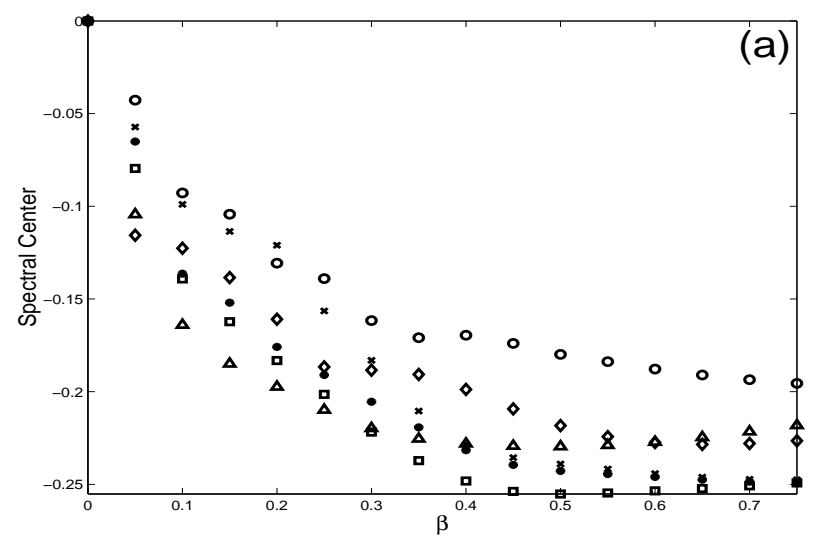

Fig. 13. The HONLS equation with nonlinear damping $0<\beta<$ $0.75, u_{0}=a(1+0.01 \cos \mu x), 0.57<a<0.67$, (a) spectral center $k_{\mathrm{m}}$ at $t=200$, (b) $\max _{t \in[0,200]} S(t)$, (c) number of rogue waves, and (d) time of last rogue wave as a function of $\beta$. The solid curves represent the averages.
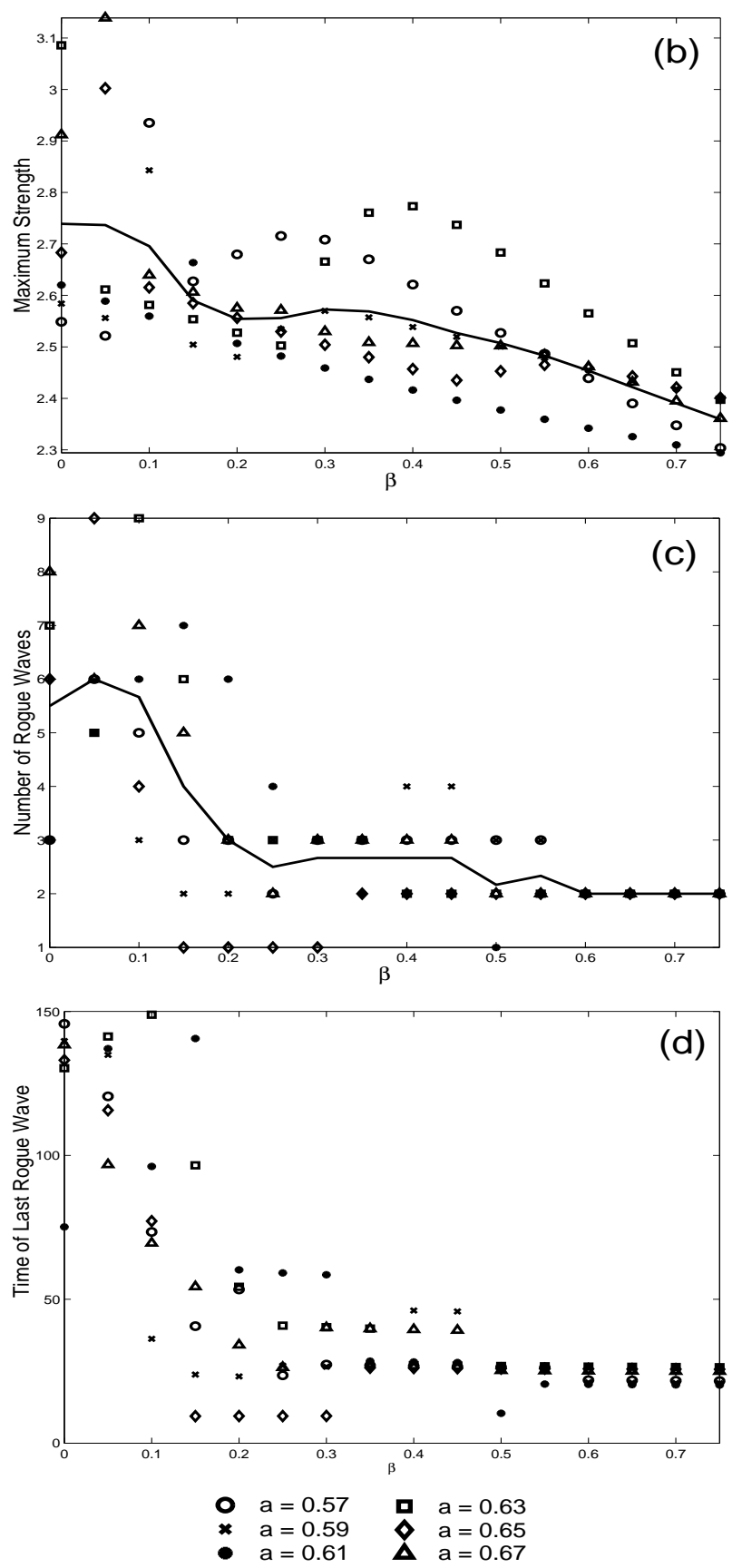

Fig. 13. Continued.

\section{Damped random oceanic sea states}

In this section we examine the generation of rogue waves in the presence of damping for sea states characterized by the Joint North Sea Wave Project (JONSWAP) spectrum:

$S(f)=\frac{\alpha g^{2}}{(2 \pi f)^{5}} e^{-\frac{5}{4}\left(\frac{f_{0}}{f}\right)^{4}} \gamma^{e^{-\frac{1}{2}\left(\frac{f-f_{0}}{\sigma f_{0}}\right)^{2}}}, \quad \sigma=\left\{\begin{array}{l}0.07 f \leq f_{0} \\ 0.09 f>f_{0}\end{array}\right.$

Here $f$ is spatial frequency, $f_{n}=k_{n} / 2 \pi, f_{0}$ is the dominant 

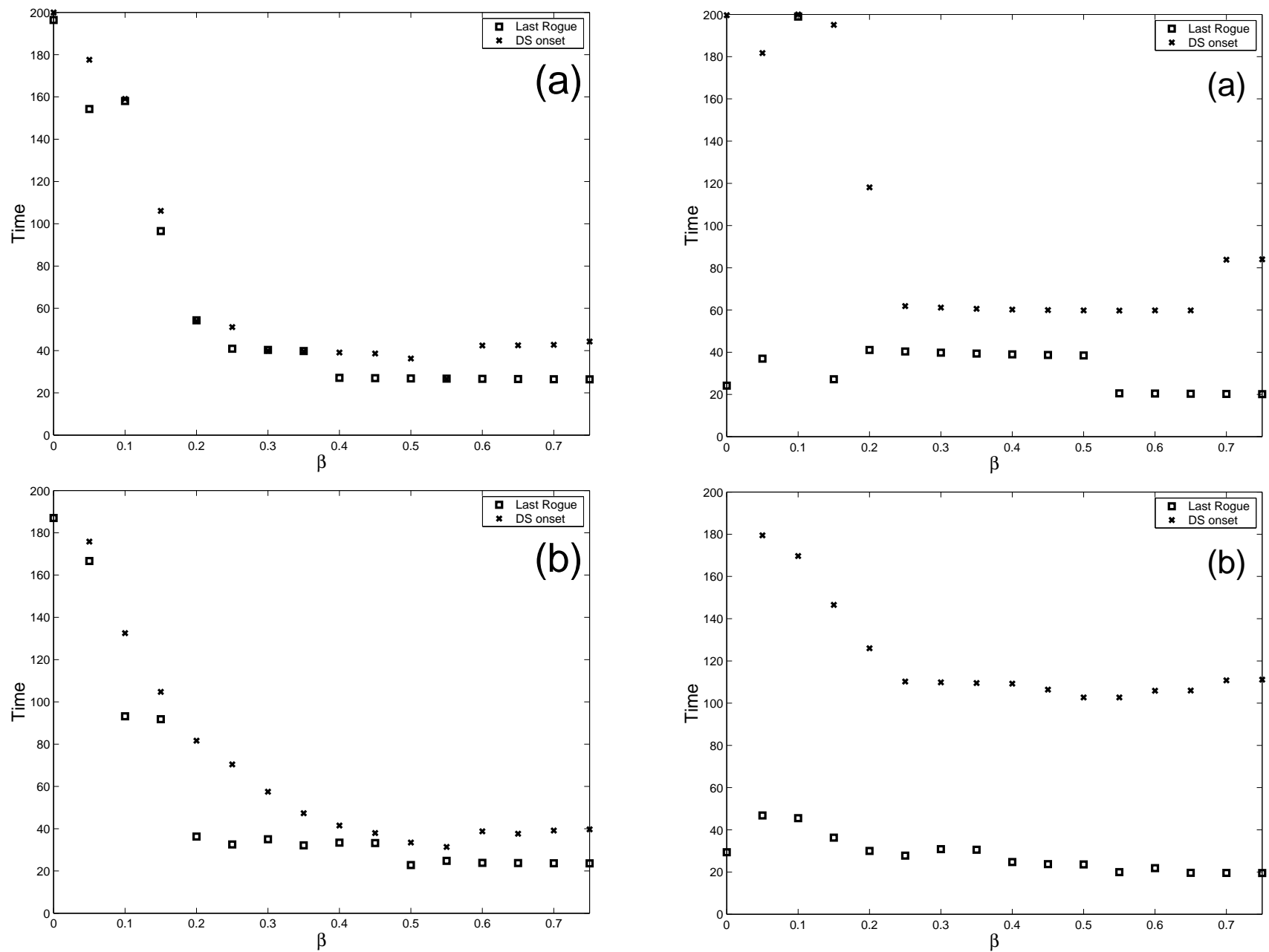

Fig. 14. The nonlinear damped HONLS equation with $\epsilon=$ $0.05, \Gamma=0$ and $0<\beta<0.75$. The time downshifting is irreversible (x) and the time the last rogue wave occurs (box) as a function of $\beta$, $u_{0}=a(1+0.01 \cos \mu x)$, where (a) $a=0.63$ and (b) averaged over $0.57<a<0.67$.

frequency determined by the wind speed at a specified height above the sea surface and $g$ is gravity. Developing storm dynamics are governed by this spectrum for a range of parameters $\gamma$ and $\alpha$. Here $\gamma$ is the peakedness parameter and $\alpha$ is related to the amplitude and energy content. The putative region of validity for the NLS equation and its higher order generalizations is $2<\gamma<8$ and $0.008<\alpha<0.02$

The initial data for the surface elevation is of the form (Onorato et al., 2001)

$\eta(x, 0)=\sum_{n=1}^{N} C_{n} \cos \left(k_{n} x-\phi_{n}\right)$,

where $k_{n}=2 \pi n / L$, the random phases $\phi_{n}$ are uniformly distributed on $(0,2 \pi)$ and the spectral amplitudes $C_{n}=$ $\sqrt{S\left(f_{n}\right) / 2 L}$. The relation of $\eta$ to the solution of the nonlinear Schrödinger equation $u(x, t)$ is given by $\eta=\frac{1}{\sqrt{2} k} \operatorname{Re}\left\{i u e^{i k x}\right\}$.

Fig. 15. The damped HONLS eqn. with $\epsilon=0.05, \Gamma=0.005$ and $0<\beta<0.75$. The time downshifting is irreversible ( $\mathrm{x}$ ) and the time the last rogue wave occurs (box) as a function of $\beta$ and $\Gamma=0.005$, $u_{0}=a(1+0.01 \cos \mu x)$, where (a) $a=0.63$ and (b) averaged over $0.57<a<0.67$.

\subsection{Nonlinear spectral diagnostic for determining rogue waves}

In previous work we proposed a nonlinear spectral decomposition of the JONSWAP data and introduced the splitting distance between two simple points, $\delta\left(\lambda_{+}, \lambda_{-}\right)=\left|\lambda_{+}-\lambda_{-}\right|$ to measure the proximity to homoclinic data of the NLS equation (Islas and Schober, 2005; Schober, 2006). Briefly, homoclinic solutions arise as an appropriate degeneration of a finite gap solution, i.e. as $\delta\left(\lambda_{+}, \lambda_{-}\right) \rightarrow 0$ the resulting double point is complex. Such a potential possesses no linear unstable modes (simple points and real double points are in general associated with neutrally stable modes), although the solution is "close" in the spectral sense to an unstable solution. Consequently, $\delta$ measures the proximity in the spectral plane to complex double points and their 

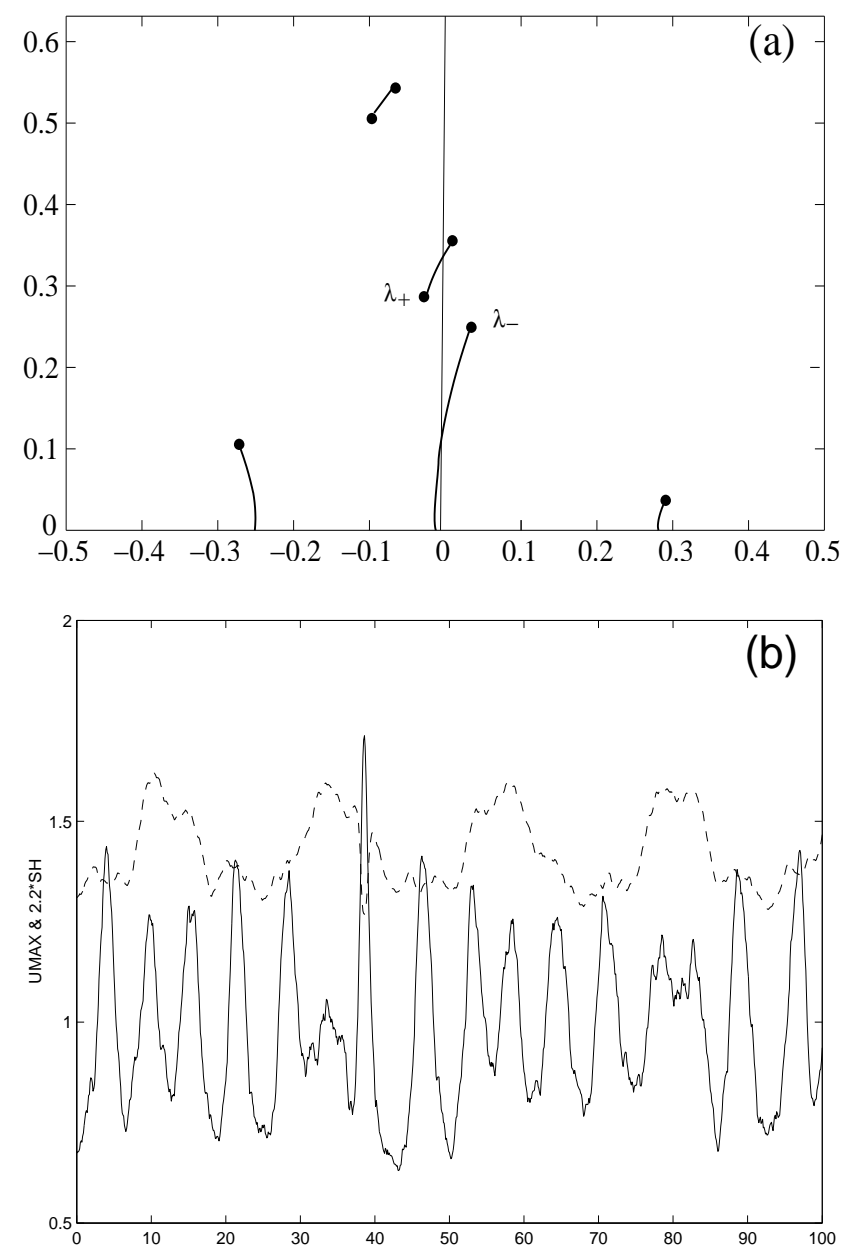

Fig. 16. (a) Spectrum and (b) evolution of $U_{\max }$ "near" to homoclinic data.

corresponding instabilities and can be used as a criterium for predicting the strength and occurrence of rogue waves (Islas and Schober, 2005).

We begin by determining the nonlinear spectrum of JONSWAP initial data given by (20) for combinations of $\alpha=0.008,0.012,0.016,0.02$, and $\gamma=1,2,4,6,8$. For each such pair $(\gamma, \alpha)$, fifty simulations were performed, each with a different set of randomly generated phases. As expected, the spectral configuration depends on the energy $\alpha$ and the peakedness $\gamma$. However, the phase information is just as important to the final determination of the spectrum. Typical examples of the numerically computed nonlinear spectrum for JONSWAP initial data $(\gamma=4, \alpha=0.016)$ with two different realizations of the random phases are shown in Figs. 16a and 17a. Since the NLS spectrum is symmetric with respect to the real axis only the spectrum in the upper half complex $\lambda$-plane is displayed.
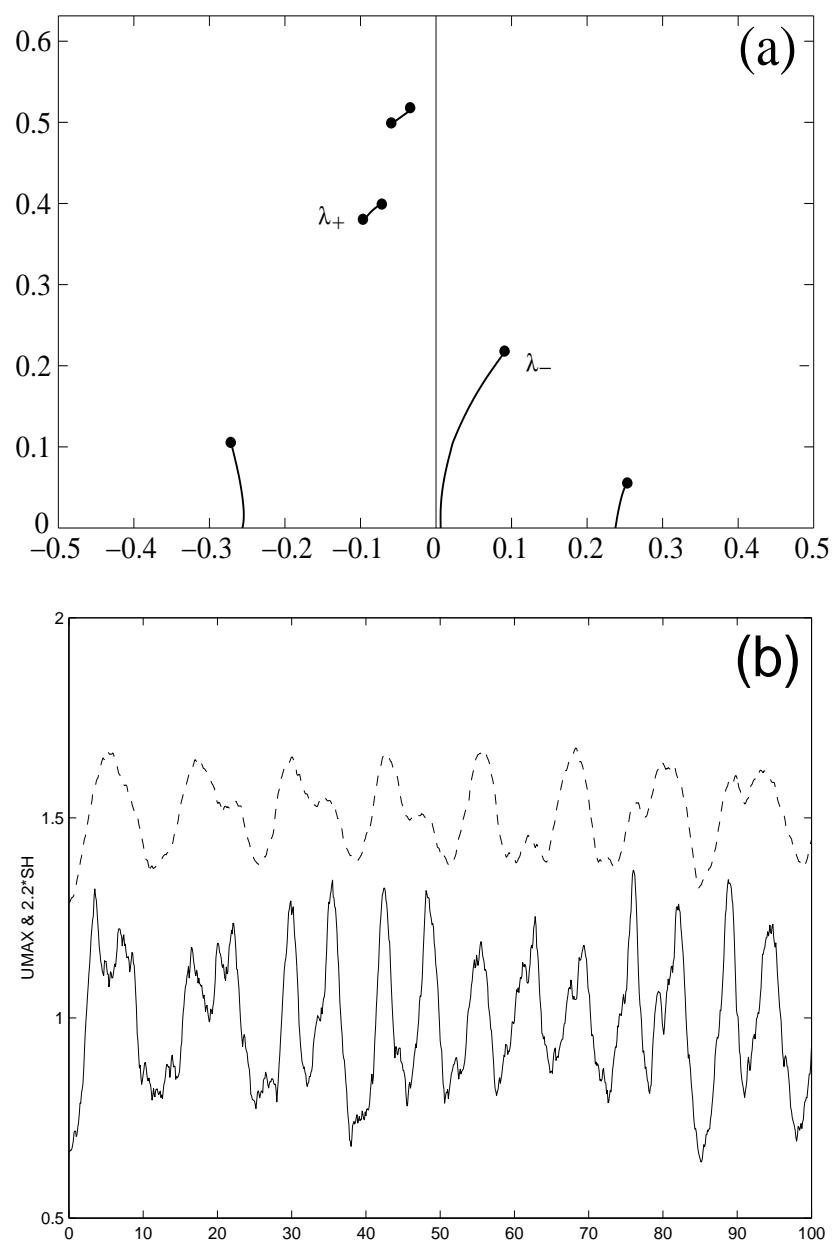

Fig. 17. (a) Spectrum and (b) evolution of $U_{\max }$ "far" from homoclinic data.

We find that JONSWAP data may correspond to "semistable" solutions, i.e. JONSWAP data can be viewed as perturbations of $\mathrm{N}$-phase solutions with one or more unstable modes (compare Fig. 16a with Fig. 1a, the spectrum of an unstable N-phase solution). In Fig. 16a the splitting distance $\delta\left(\lambda_{+}, \lambda_{-}\right) \approx 0.07$, while in Fig. $17 \mathrm{a} \delta\left(\lambda_{+}, \lambda_{-}\right) \approx$ 0.2 . Thus, for fixed $\alpha$ and $\gamma$ as the phases are randomly varied, the spectral distance $\delta$ of typical JONSWAP data from homoclinic data varies significantly and can be quite "near" as in Fig. 16a, or "far" from homoclinic data as in Fig. 17a.

The most striking feature is that irrespective of the values of $\alpha$ and $\gamma$, in simulations of the NLS equation (10) extreme waves develop for JONSWAP initial data that is "near" NLS homoclinic data, whereas the JONSWAP data that is "far" from NLS homoclinic data typically does not generate extreme waves. Figures $16 \mathrm{~b}$ and $17 \mathrm{~b}$ show the corresponding evolution of $U_{\max }\left(U_{\max }=\max _{x \in[0, L]}|u(x, t)|\right)$, obtained with the NLS equation. $U_{\max }$ is given by the solid curve and as a reference the threshold for a rogue wave, 2.2 times 

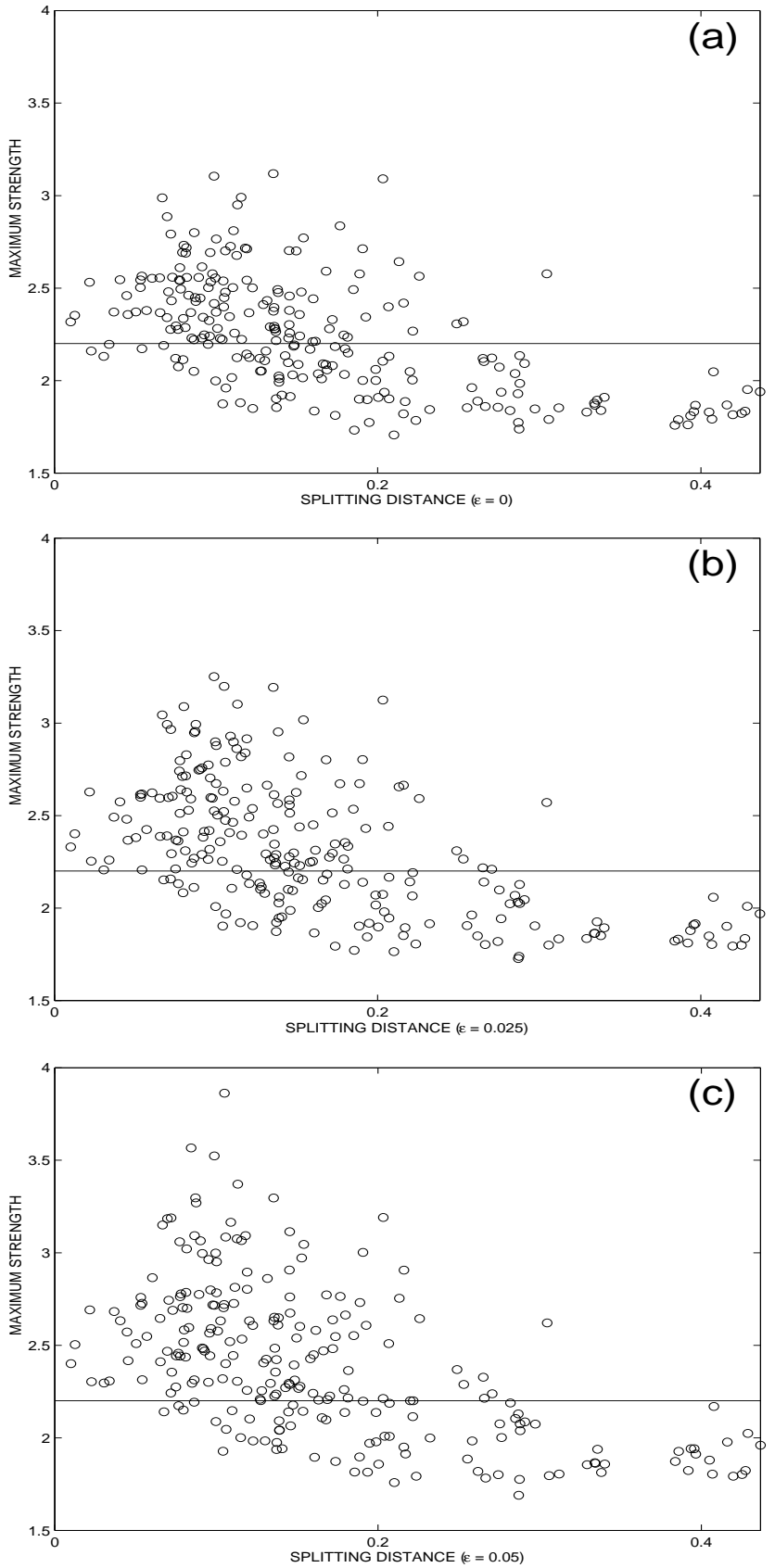

Fig. 18. Maximum strength versus the splitting distance $\delta\left(\lambda_{+}, \lambda_{-}\right)$ for (a) the NLS equation and the HONLS equation with (b) $\epsilon=0.025$, and (c) $\epsilon=0.05$.

the the significant wave height, $2.2 H_{\mathrm{s}}$, is given by the dashed curve. Figure $16 \mathrm{~b}$ shows that when the nonlinear spectrum is near homoclinic data, $U_{\max }$ exceeds $2.2 H_{\mathrm{s}}$ (a rogue wave develops at $t \approx 40$ ). Figure $17 \mathrm{~b}$ shows that when the nonlinear spectrum is far from homoclinic data, $U_{\max }$ is significantly below $2.2 H_{\mathrm{s}}$ and a rogue wave does not develop. As a result we can correlate the occurrence of rogue waves characterized by JONSWAP spectrum with the proximity to homoclinic solutions of the NLS equation.

\subsection{JONSWAP experiments with the NLS and HONLS equations}

The results of hundreds of simulations of the NLS and HONLS equations consistently show that proximity to homoclinic data is a crucial indicator of rogue wave events. Figure 18 provides a synthesis of 250 random simulations of (a) the NLS equation and of $(b-c)$ the HONLS equation $(\beta=\Gamma=0)$ for $\epsilon=0.025$ and $\epsilon=0.05$, respectively, using JONSWAP initial data for different $(\gamma, \alpha)$ pairs with $\gamma=$ $1,2,4,6,8$ and $\alpha=0.008,0.012,0.016,0.02$ and randomly generated phases. Our considerations are restricted to semi-stable N-phase solutions near to unstable solutions of the NLS with one UM. Each circle represents the maximum strength, $\max _{t \in[0,20]} S(t)$, attained in time during one simulation, as a function of the splitting distance $\delta\left(\lambda_{+}, \lambda_{-}\right)$. A horizontal line at $U_{\max } / H_{\mathrm{s}}=2.2$ indicates the reference strength for rogue wave formation. We identify two critical values $\delta_{1}(\epsilon)$ and $\delta_{2}(\epsilon)$ that clearly show that (i) if $\delta<\delta_{1}$ (near homoclinic data) the likelihood of a rogue wave is extremely high; (ii) if $\delta_{1}<\delta<\delta_{2}$, the likelihood of obtaining rogue waves decreases as $\delta$ increases and, (iii) if $\delta>\delta_{2}$ the likelihood of a rogue wave occurring is extremely small.

As $\alpha$ and $\gamma$ are varied the behavior is robust. The maximum wave strength and the occurrence of rogue waves are well predicted by the proximity to homoclinic solutions. The individual plots of the strength vs. the splitting distance $\delta$ for particular pairs $(\gamma, \alpha)$ are qualitatively the same regardless of the pair chosen. Comparing Fig. 18a-b we find that enhanced focusing and increased wave strength occur in the chaotic HONLS evolution as compared with the NLS evolution. Figure 18 shows that as the perturbation strength $\epsilon$ increases, the maximum wave strength and the likelihood of rogue waves occuring in a given simulation increases.

\subsection{JONSWAP experiments with the damped HONLS equations}

In this section we show that, in the presence of damping, the nonlinear spectrum and the proximity to homoclinic data is likewise an important indicator of the maximum strength in time and likelihood of rogue waves.

Figure 19 shows the maximum wave strength vs. the splitting distance $\delta\left(\lambda_{+}, \lambda_{-}\right)$for 250 random simulations of the damped HONLS equation $(\epsilon=0.05)$, with either (a) linear damping, $\beta=0, \Gamma=0.025$, or with (b) nonlinear damping $\beta=0.5, \Gamma=0$. As before, JONSWAP initial data was used for different $(\gamma, \alpha)$ pairs with $\gamma=1,2,4,6,8$ and $\alpha=0.008,0.012,0.016,0.02$ and randomly generated phases. Each circle represents the maximum strength attained during one simulation, $0<t<20$. 

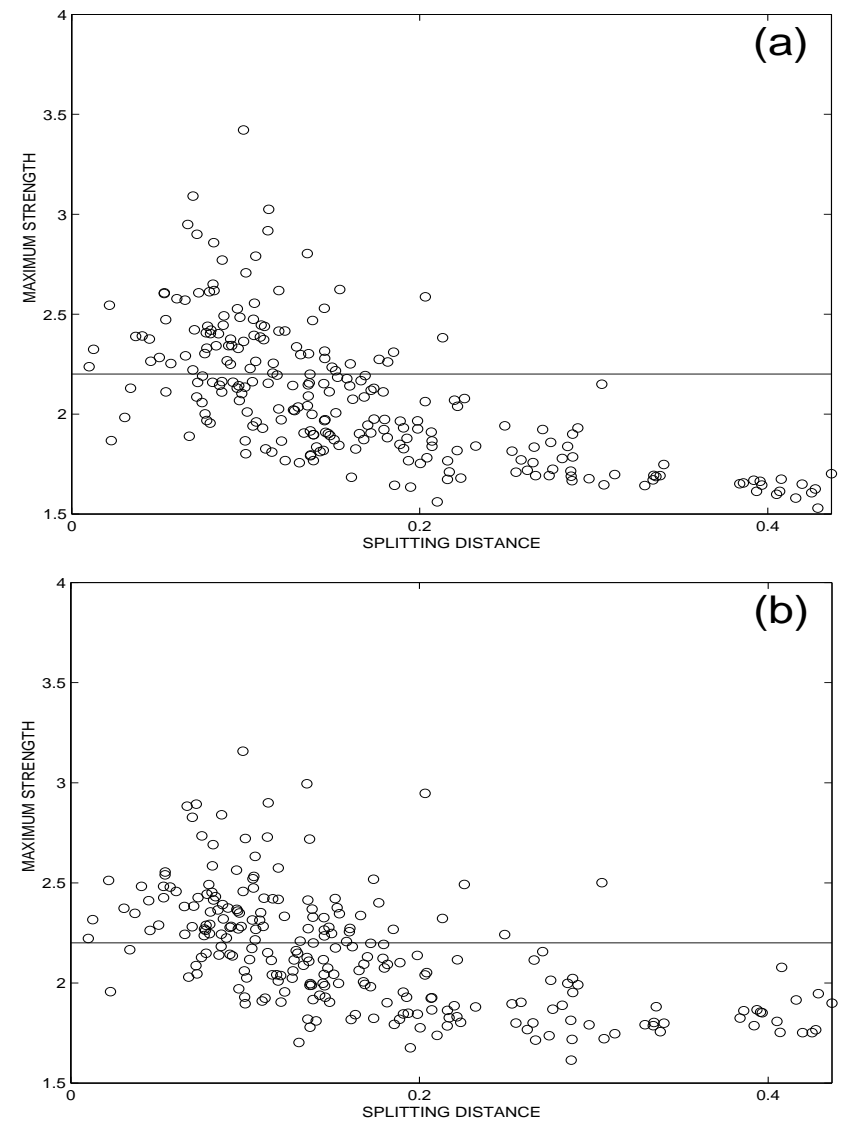

Fig. 19. Maximum strength vs. splitting distance $\delta\left(\lambda_{+}, \lambda_{-}\right)$for the HONLS equation $(\epsilon=0.05)$ with either (a) linear damping, $\beta=0$, $\Gamma=0.025$, or (b) nonlinear damping $\beta=0.5, \Gamma=0$.

In Fig. 19 it is evident that the strength and likelihood of rogue waves occuring in a given simulation is typically smaller when damping is present (compare to results of the HONLS equation, Fig. 18c). A cutoff vaue of $\delta$ exists such that rogue waves typically do not occur if $\delta>\delta_{\text {cutoff. We }}$ determine $\delta_{\text {cutoff }}$ by requiring that $95 \%$ of the rogue waves occur for $\delta<\delta_{\text {cutoff. }}$ For example, the cutoff values for the linear $(\Gamma=0.02)$ and nonlinear $(\epsilon \beta=0.02)$ damped HONLS equation are $\delta_{\text {cutoff }}^{\text {damped }} \approx 0.16,0.17$, respectively, which are less than the cutoff value for the undamped HONLS equation where, $\delta_{\text {cutoff }}^{\text {undamped }} \approx 0.2$. Significantly, this implies that when damping is present the JONSWAP initial data must be closer to homoclinic data and instabilities to obtain rogue waves.

Figure 20 shows $\delta_{\text {cutoff }}^{\text {damped }}$ as a function of the damping parameters $\Gamma$ (circles) and $\epsilon \beta$ (boxes). For each of the ten values of $\Gamma$ and of $\epsilon \beta, 0<\Gamma, \epsilon \beta<0.04,250$ simulations of the damped HONLS equation were carried out. We find that $\delta_{\text {cutoff }}^{\text {damped }}$ is generally decreasing as the strength of the damping increases. The cutoff value in $\delta$ for the nonlinearly damped case is not monotonically decreasing as atypical cases exist where a small increase in damping may be

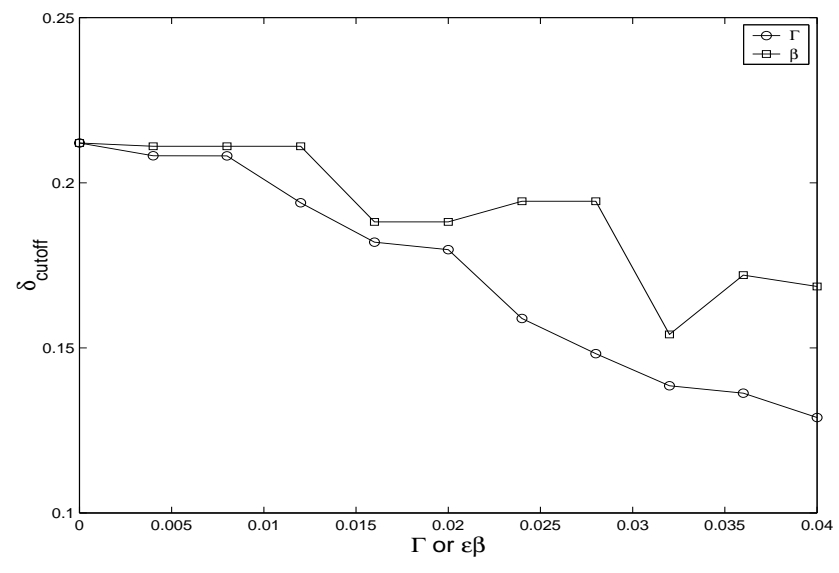

Fig. 20. The damped HONLS equation $(\epsilon=0.05)$ : $\delta_{\text {cutoff }}^{\text {damped }}$ as a function of $\Gamma$ (circles) and $\epsilon \beta$ (boxes).

offset by a coalescence of the modes due to changes in the focusing times. The overall decay in $\delta_{\text {cutoff }}^{\text {damped }}$ indicates that for rogue waves to occur the JONSWAP initial data must lie in a shrinking neighborhood of the homoclinic data. Thus the proximity to instabilities and homoclinic data is more essential for the development of rogue waves when damping is present.

The observations from the nonlinear spectral diagnostic complement the earlier results characterizing the nonlinear damped evolution. In Sect. 4, the numerical simulations start very close to homolinic data (for initial data (19) the splitting distance is on the order of $10^{-6}$, an effective rogue wave regime). The first pair of rogue waves were not significantly altered by the damping. However after permanent downshifting occured any further rogue waves were inhibited. Here we are generalizing to JONSWAP initial data which are not necessarily close to homoclinic data. We are monitoring the maximum strength in time as a function of $\delta$ which is a separate issue from the total number of rogue waves in a given simulation.

Finally we remark that we recently examined rogue waves and downshifting in the damped Dysthe equation (Islas and Schober, 2010). While the Dysthe equation does not conserve momentum, we have obtained qualitatively similar results. For the nonlinearly damped Dysthe equation, irreversible downshifting also occurs for all values of $\beta$, although on a slightly longer timescale. Rogue waves do not occur in the time series after the downshifting is permanent. Similarly, $\delta_{\text {cutoff }}^{\text {damped }}$ generally decreases as the strength of the damping increases suggesting the heightened importance of proximity to homoclinic data when obtaining rogue waves in the damped Dysthe regime.

Acknowledgements. This work was partially supported by NSF Grant \# NSF-DMS0608693. 
Edited by: C. Kharif

Reviewed by: two anonymous referees

\section{References}

Ablowitz, M. J., Hammack, J., Henderson, D., and Schober, C. M.: Modulated Periodic Stokes Waves in Deep Water, Phys. Rev. Lett., 84, 887-890, 2000.

Ablowitz, M. J., Hammack, J., Henderson, D., and Schober, C. M.: Long time dynamics of the modulational instability of deep water waves, Physica D, 152-153, 416-433, 2001.

Ablowitz, M. J. and Segur, H.: Solitons and the Inverse Scattering Transform, SIAM, 1981.

Calini, A., Ercolani, N. M., McLaughlin, D. W., and Schober, C. M.: Mel'nikov analysis of numerically induced chaos in the nonlinear Schrödinger equation, Physica D, 89, 227-260, 1996.

Calini, A. and Schober, C. M.: Homoclinic chaos increases the likelihood of rogue waves, Phys. Lett. A, 298, 335-349, 2002.

Cox, S. M. and Matthews, P. C.: Exponential time differencing for stiff systems, J. Comput. Phys., 176, 430-455, 2002.

Dysthe, K.: Note on a modification to the nonlinear Schrodinger equation for deep water, Proc. R. Soc. Lon. Ser.-A, 369, 105114, 1979.

Ercolani, N., Forest, M. G., and McLaughlin, D. W.: Geometry of the Modulational Instability Part III: Homoclinic Orbits for the Periodic Sine-Gordon Equation, Physica D, 43, 349-384, 1990.

Gramstad, O. and Trulsen, K.: Hamiltonian form of the modified nonlinear Schrödinger equation for gravity waves on arbitrary depth, J. Fluid Mech., in press, 2011.

Hammack, J. L., Henderson, D. M., and Segur, H.: Deep-water waves with persistent, two-dimensional surface patterns, J. Fluid Mech., 532, 1-51, 2005.

Hara, T. and Mei, C. C.: Frequency down-shift in narrowbanded surface waves under the influence of wind, J. Fluid Mech., 230, 429-477, 1991.

Hara, T. and Mei, C. C.: Wind effects on the nonlinear evolution of slowly varying gravity-capillary waves, J. Fluid Mech., 267, 221-250, 1994.

Henderson, K. L., Peregrine, D. H., and Dold, J. W.: Unsteady water wave modulations: fully nonlinear solutions and comparison with the nonlinear Schrödinger equation, Wave Motion, 29, 341$361,1999$.

Huang, N., Long, S., and Shen, Z.: The mechanism for frequency downshift in nonlienar wave evolution, Adv. Appl. Mech., 32, 59-117, 1996.

Islas, A. and Schober, C. M.: Predicting rogue waves in random oceanic sea states, Phys. Fluids, 17, 1-4, 2005.

Islas, A. and Schober, C. M.: Rogue waves, dissipation, and downshifting, Physica D, submitted, 2010.

Kato, Y. and Oikawa, M.: Wave number down-shift in Modulated Wavetrain through a Nonlinear Damping effect, J. Phys. Soc. Jpn, 64, 4660-4669, 1995.
Khaliq, A. Q. M., Martin-Vaquero, J., Wada, B.A., and Yousuf, M.: Smoothing Schemes for Reaction-Diffusion Systems with Nonsmooth Data, J. Comput. Appl. Math. 223, 374-386, 2009.

Kharif, C. and Pelinovsky, E.: Focusing of nonlinear wave groups in deep water, JETP Lett., 73, 170-175, 2001.

Kharif, C. and Pelinovsky, E.: Physical mechanisms of the rogue wave phenomenon, Eur. J. Mech. B-Fluid., 22, 603-634, 2004.

Krasitskii, V.: On reduced equations in the Hamiltonian theory of weakly nonlinear surface waves, J. Fluid Mech., 272, 1-20, 1994.

Lake, B., Yuen, H., Rungaldier, H., and Ferguson, W.: Nonlinear deep-water waves:theory and experiment. Part 2. Evolution of a continuous wave train, J. Fluid Mech., 83, 49-74, 1977.

Matveev, V. B. and Salle, M. A.: Darboux Transformations and Solitons, Springer-Verlag, 1991.

McLaughlin, D. W. and Schober, C. M.: Chaotic and homoclinic behavior for numerical discretizations of the nonlinear Schrodinger equation, Physica D, 57, 447-465, 1992.

Onorato, M., Osborne, A., Serio, M., and Bertone, S.: Freak wave in random oceanic sea states, Phys. Rev. Lett., 86, 5831-5834, 2001.

Osborne, A., Onorato, M., and Serio, M.: The nonlinear dynamics of rogue waves and holes in deep-water gravity wave trains, Phys. Lett. A, 275, 386-393, 2000.

Schober, C. M.: Melnikov analysis and inverse spectral analysis of rogue waves in deep water, Eur. J. Mech. B-Fluids, 25(5), 602620, doi:10.1016/j.euromechflu.2006.02.005, 2006.

Segur, H., Henderson, D., Carter, J., Hammack, J., Li, C., Pheiff, D., and Socha, K.: Stabilizing the Benjamin-Feir Instability, J. Fluid Mech., 539, 229-271, 2005.

$\mathrm{Su}, \mathrm{M}$.: Evolution of groups of gravity waves with moderate to high steepness, Phys. Fluids, 25, 2167-2174, 1982.

Trulsen, K. and Dysthe, K.: Frequency down-shift through self modulation and breaking, in: Water Wave Kinematics, edited by: Torum, A. and Gudmestad, O. T., Kluwer 561-572, 1990.

Trulsen, K. and Dysthe, K.: Frequency down-shift in threedimensional wave trains in a deep basin, J. Fluid Mech., 352, 359-373, 1997a.

Trulsen, K. and Dysthe, K.: Freak waves - a three dimensional wave simulation, in: Proceedings of the 21st Symposium on Naval Hydrodynamics, edited by: Rood, E. P., Nat. Acad. Press, 550 560, 1997 b.

Uchiyama, Y. and Kawahara, T.: A possible mechanism for frequency down-shift in nonlinear wave modulation, Wave Motion, 20, 99-110, 1994.

Zakharov, V. E. and Shabat, A. B. Exact theory of two-dimensional self-focusing and one-dimensional self-modulation of waves in nonlinear media, Sov. Phys. JETP, 34, 62-69, 1972. 